\title{
A Fast Spatial Scanning Combination Emissive and Mach Probe for Edge Plasma Diagnosis
}

\author{
R.D. Lehmer, B. LaBombard, R.W. Conn
}

UCLA-PPG-1228 Aprìl, 1989

\section{DISCLAIMER}

\begin{abstract}
This repon was prepared as an account of work sponsored by an agency of the Uniled States Government. Neither the United States Government nor any agency thereof, nor any of their employees, makes any warranty, express or implied, or assumes any iegul liability or responsibility for the accuracy, completeness, or usefulness of any information, apparatus, product, or prosess disclosed, or represents that its use would not infringe privately owned rights. Reference hetein to any specific commercial product, process, or service by trade name, trademark. manufacturer, of otherwise does not necessarily constitute or imply its endorsemeat, recommendation, or favorifig by the United States Government or any agency thereof. The views and opinions of authors expressed herein do not necessarily state or reffect those of the United States Government or any agency thereof.
\end{abstract}

Institute of Plasma and Fusion Research

Department of Mechanical, Aerospace, and Nuclear Engineering

University of Califumia, Los Angeles

Los Angeles, CA, 90024, U.S.A. 


\section{DSCLARER}

This repon was propared as an exenent of work sponsorrd by an atency of the United Stmes Goverument. Neither the United Suter Government nor wy escacy thereof, nor sny of their

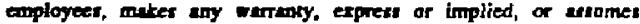
any legal liability or responsibility for the accaracy, completeness. of esefulness of any information, apparatus. produch or process ditelosed, of reprevente that its ve would not infringe privutely owned rights. Refurence herein to any specific conmercial produrt process, or service by trade name, uedemerk, manufucturer, or otherwise, does not necessurily conntituee of imply its endotuement, recommendation. or favotins by the United States Government of any agency thereof. The views and opinions of anthors expressed lierein do not nesesumily state of reflect those of the United Stures Govemment or any asency thereof. 


\title{
A Fast Spatial Scanning Combination Emissive and Mach Probe for Edge Plasma Diagnosis
}

\author{
by \\ R. D. Lehmer, B. LaBombard ${ }^{1}$, R. W. Conn \\ Institute of Plasma and Fusion Research \\ Department of Mechanical, Aerospace, and Nuclear Engineering \\ University of California, Los Angeles \\ Los Angeles, California 90024 \\ IPresent address: \\ Plasma Fusion Center \\ Massachusetts Institute of Technology \\ Cambridge, Massachusetts 02139
}

\begin{abstract}
A fast spatially scanning emissive and mach probe has been developed for the measurement of plasma profiles in the PISCES facility at UCLA. A pneumatic cylinder is used to drive a multiple tip probe along a $15 \mathrm{~cm}$ stroke in less than $400 \mathrm{msec}$, giving single shot profiles while limiting power deposition to the probe. A differentially pumped sliding $\mathrm{O}$-ring seal allows the probe to be moved between shots to infer two and three dimensional profiles. The probe system has been used to investigate the plasma potential, density, and parallel mach number profiles of the presheath induced by a wall surface and scrape-off-layer profile modifications in biased limiter simulation experiments. Details of the hardware, data acquisition electronics, and tests of probe reliability are discussed.
\end{abstract}


Various mach probe theories are reviewed and the resulting mach number profiles from these theories are examined critically. Typical plasma profiles demonstrating the utility of the diagnostic for studying tokamak-like edge plasmas will also be presented. Additional techniques for improving scanning emissive probe diagnostics are proposed. Work Supported by U.S. DOE Contract No. DE-FG03-86ER52134. 


\section{Table of Contents}

Section

page

I. Introduction. . . . . . . . . . . . . . . . . .

II. Principle of Operation of Scanning Probe. ............

A. Mechanical Design. ................. 5

B. Electronics and Control. . . . . . . . . . . . . .

III. The Floating and Space Potential and the Role of Sheaths on Their Measurement. . . . . . . . . . . . . . . . . .9

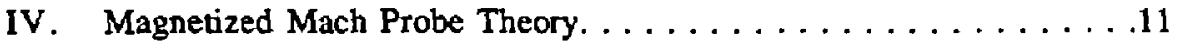

v. Probe Emission Testing. . . . . . . . . . . . . . . 24

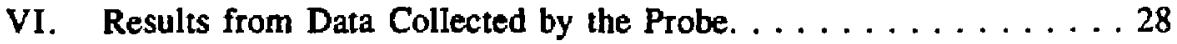

VII. Future Diagnostic Development. . . . . . . . . . . . . . 40

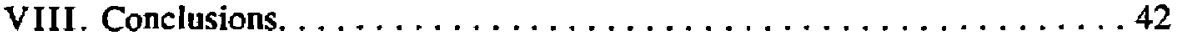

IX. Acknowledgements. . . . . . . . . . . . . . 43

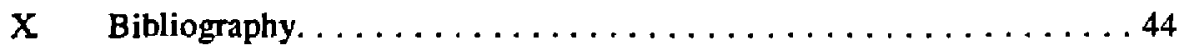




\section{Introduction}

The study of the physics of presheaths and tokamak-like scrape-off-layers in the PISCES plasma-surface interaction facility 1 has lead to the development of a fast injection, spatially scanning combination emissive/mach probe diagnostic. The emissive probe is a unique diagnostic tool because it permits the simple, continuous monitoring of the space potential 2,3 as long as the probe can emit more electrons than the incident electron flux from the plasma. Other designs 4.5 circumvent the condition of strong emission through the use of secondary electron emission and feedback electronics.

Fast spatially scanning probe diagnostics are very important and unique tools used in analyzing tokamak edge plasmas. Profiles of potential, density, temperature, parallel mach number, and heat flux, as well as the fluctuations of the first three quantities, can be accumulated simultaneously to characterize the transport in the edge of a tokamak plasma. Profiles from a scanning probe generally have better spatial resolution than non-perturbing optical or RF edge diagnostics that typically rely on mathematical tools such as the Abel inversion to infer profile shapes from the raw data. A fast spatially scanning probe can be used several times during a single tokamak shot to observe temporal changes in the profiles and transport. The same diagnostic can also be used to observe edge profile modifications due to biased limiter or divertor structures in the plasma. 
The combination emissive and mach probe tip is driven across the plasma by a pneumatic cylinder, which allows for single scan or shot profiles of the space or floating potential, density, and parallel mach number. Since the PISCES facility has a steady state plasma generator, profiles of the space and floating potentials under identical plasma conditions can be obtained by using successive shots of the fast probe. During the first shot, the emissive probe tip is left cold in order to record the floating potential. Before the second shot, the probe is resistively heated so that it will thermionically emit electrons into the plasma and electrically float at the space potential by cancelling the effect of the sheath in front of the probe.

The PISCES experimental facility is used to simulate plasmasurface interactions occurring at the edge of fusion confinement experiments (figure 1). The plasma is produced by a radiatively heated lanthanum hexaboride cathode supplying primary electrons to a reflex arc discharge. The electrons in the plasma are radially confined by a DC solenoid magnetic field of 200 to 1400 gauss and are lost mostly by recombination with the ions at the endplates. Steady state plasma densities of $10^{11}$ to $10^{13} \mathrm{~cm}^{-3}$ with electron temperatures of 5 to $30 \mathrm{eV}$ are typically achieved. The typical operating neutral pressures in the main chamber are between $10^{-4}$ to $10^{-3}$ torr, while the neutral pressure in the source region can be a few millitorr. These values correspond to typical conditions in the edge plasma of tokamaks. The ions have been observed to have a 
temperature of less than $1 \mathrm{eV}$. A much more detailed explanation of the experimental arrangement is given in another reference 6.

This paper will cover the theory, design, and operation of the PISCES scanning emissive/mach probe diagnostic and its data acquisition electronics. Representative profiles of potential, density, and parailel mach number measured in the PISCES plasma will be presented. Various mach probe theories will be compared using the mach probe data collected. Evidence for a double sheath forming around the emissive probe tip is presented. Finally, techniques for eliminating the error induced by this effect in inferring the space potential are discussed.

\section{Principle of Operation of Scanning Probe}

The diagnostic is designed to give single shot spatial profiles of the potential, density, and parallel mach number. The diagnostic infers these parameters through the use of five electrical probes protruding from the end of a cylindrical ceramic rod. A thoriated tungsten wire loop emissive probe records the floating or space potential of the plasma depending on whether or not the probe is heated into strong emission. The dexsity and parallel mach number profiles are computed from the ion saturation current profiles of the four other probes, which are grouped into pairs. For each pair of probe tips, one probe faces towards the plasma source end of the experiment ("upstream"), while the other faces the opposite direction 
("downstream"). The probe tip structure is designed so that there is no direct electrical connection of probe tips along the field lines.

One pair of probes has dimensions physically larger than the ion Larmor radius in the plasma. In this case, the probe tip pair can be referred to as a magnetized mach probe. By recording the difference in the flux incident on the upstream and downstream magnetized probes, the parallel mach number profile can be inferred. A density profile can also be computed by looking at the total current collected by both the up and downstream probes.

The tips of the other pair of collecting probes are physically smaller than the magnetized pair, and depending upon the axial magnetic field strength, they can be smaller than the ion Larmor radius. These probes are used to study magnetized versus unmagnetized probe theory and wakes downstream of objects inserted into the plasma7.

In order to measure the space potential profile, the emissive probe is heated to its equilibrium temperature before the probe is inserted into the plasma. This ensures that the probe is in a regime of space charge limited emission rather than temperature limited emission. Monitoring the space potential by temperature limited emission requires a more complicated sheath criterion than space charge limited emission.

When a spatial profile is recorded, the position of the probe, the potential of the emissive probe, and the ion saturation current 
for the four mach probe tips are all fed through isolation amplifiers to a CAMAC data logger. The CAMAC then transfers the digitized da:a to a MicroVaxII, which handles the database and computes and displays the raw and processed data.

\section{A. Mechanical Design}

Since the residence time of the fast probe tip in the plasma is short, no active water cooling of the structure is necessary. Thus, the physical dimensions of the probe can be much smaller than its actively cooled counterpart, and the probe will be only a small perturbation to the plasma.

The basic design of the probe is shown in figure 2. The probe shaft is made of an alumina rod with six holes accommodating the tip wiring. The probe tip itself consists of an emissive probe and two pairs of collection probes, each pair featuring upstream and downstream probes. The magnetized collection probes are simply .030" diameter molybdenum wires that are shadowed from each other by the chisel point of the alumina insulator of the probe. The pair of small collecting probes are made of .010" diameter tungsten wire, also insulated by small alumina tubing.

The emissive probe construction is slightly more complicated. Originally, the emissive probe was built entirely of $.010^{\prime \prime}$ diameter thoriated tungsten wire slipped through the holes of the alumina tubing and potted to the aluminat with high temperature ceramic 
adhesive. The attachment of the tungsten wire to the insulator is very important in this design because the thermal inertia of the alumina rod keeps the tungsten in the insulator cooler than the tip. Since the specific resistance of tungsten versus temperature increases an order of magnitude between room temperature and $3000^{\circ} \mathrm{K}$, most of the power will be deposited in the tip. However, this design was not entirely successful because hot spots were created at the joints of the sections of alumina. Thus, mosi of the heating power was lost before reaching the tip, and the tip rever reached the temperature required for space charge limited emission in the PISCES plasma.

To alleviate this problem, a revised design has been used whereby a thoriated tungsten tip is brazed to $.030^{-1}$ dianster copper leads that feed the electrical heating powar through the insulator. The alumina rod stiil plays an important role, however, in that it acts as a heat sink to keep the braze and copper sool when the probe tip is heated.

Figure 3 shows the pneumatic actuator for the fast scanning probe. The pneumatic artuator is mountea on a robust structural member to ensure that the probe has a straight line traje:tory and the vacuum bellows is properly compressed. In addition, the Teflon spacer near the end of the tip slides inside the copper guard and redices tha vibrational motion of the tip. The pneunatic cylinder provides a relatively constant travel velocity in and out at 
$100 \mathrm{~cm} / \mathrm{sec}$ with a short zero velocity dwell time at the end of the stroke. Position sensing is done with a linear potentiometer.

When the fast scanning probe is swept through the plasma, it records profiles of the various quantities along its trajectory through the plasma. In order to provide the flexibility to scan the entire plasma volume, an X-Y table has been constructed for the fast probe assembly. The entire probe assembly is mounted to the $X-Y$ table and slides on a differentially pumped double O-ring seal. The maximum displacement of the $X-Y$ table in each direction is limited by the $8 \mathrm{~cm}$ port diameter. This is sufficient to scan nearly the entire plasma diameter in addition to a distance of $8 \mathrm{~cm}$ along the column.

\section{B. Electronics and Control}

Figure 4 shows the control electronics for the PISCES fast probe system. Since the emissive probe has to be heated for a period much longer than the sampling window of the CAMAC, there is a separate preheating and trigger circuit for the fast probe diagnostic. The preheating timer circuit is made up two independent timing circuits. The first one energizes the filament heating circuit, while the zecond timer sends the CAMAC trigger pulse and energizes the air valve solenoid on the pneumatic cylinder. In general, the timers a set so that the emissive probe is heated to its equilibrium temperature while at rsst and the heating is continued during the probe's inward 
stroke. This ensures that the probe will be in strong emission throughout the entire inward stroke.

The data acquisition electronics are shown in figure 5. The collecting probes are held at ion saturation by a DC power supply and the current drawn by each probe is measured as a voltage across a known resistance. Gigh impedance, low distortion op amp current monitors are used to isolate the probes from one another since all the probes are driven by a single power supply. The potential of the emissive probe tip is measured at the center of the resistor bridge, so that the circuit is balanced. Therefore, the emissive probe tip floats because of the transformer isolation, and the $\mathrm{AC}$ heating current is eliminated from the measurement because of the balanced resistor bridge. The measured potential is then sent through a voltage divider circuit to produce a low voltage sigral. The resistors for the current channels and the voltage divider for the potential channel are chosen so that the maximum output voltage will be 5 volts, which is the saturation level of the CAMAC.

Since the PISCES CAMAC records 2048 points at a rate of $5 \mathrm{kHz}$, the digitizing window is approximately $400 \mathrm{~ms}$. Of that, the first $100 \mathrm{~ms}$ of the digitizing window is used to record offset levels in the data channels before the probes are inserted into the plasma. The offset is subtracted from the recorded data later on.

Figure 6 is a schematic diagram of the PISCES data acquisition and analysis network. Once the raw data has been accumulated in 
the CAMAC, the PISCES node receives the raw data via the fiber optic serial highway and then stores the data in the PISCFS database. Once the raw data is in the database, the PISCES node computes transformed data that converts the stored raw voltage values from the CAMAC into the actual values of interest by applying the appropriate multiplier or function and labeling the data with the proper units. The transformed data can then be displayed with the McCool graphics package. The transformed data from a series of shots can be temporally processed and accumulated into a single file on the PISCES node for later analysis and display on the FRAN VAX machine using the NCAR graphics package.

\section{The Floating and Space Potential and the Role of Sheaths on Their Measurement}

In general, a sheath will form in front of a material surface as a response to the disparity in the ion and electron fluxes incident on the surface as the discharge forms 8 . Since the electrons move with a greater velocity than the ions, the surface will become negatively charged, thus depressing its potential and repelling electrons. When the surface is sufficiently negative such that the electron flux equals the ion flux to the surface, the potential at the surface is said to be at the floating potential. Therefore, a surface at the floating potential collects no net current from the plasma.

However, rather than measure the floating potential, one would like to measure the potential distribution in the plasma to determine 
the electric fields that are set up in there. Using a non-emitting probe to accurately determine the space potential is difficult in most practical cases. For cold probes, the space potential can be found from the knee (the transition to electron saturation current) of the I$V$ characteristic of a Langmuir probe. In many experiments, this knee is not very well defined due to several factors: 1) A nonMaxwellian electron velocity distribution affect the interpretation of the transition region of the I.V characteristic; 2) as the probe is biased near the space potential, electrons freely flow to the probe and change the local discharge conditions.

If the probe surface is allowed to emit electrons back into the plasma, the emitted electrons may cancel the sheath and allow the probe to "float" at the space potential. However, because the electrons are emitted from the probe at the filament temperature, they are moch cooler than the bulk plasma electrons. The result of this disparity is that only a fraction of the electron current entering the sheath is cancelled by emitted electron current 5 . In order for the emitting surface to draw no net current, a sheath will still have to be present to reflect the necessary fraction of bulk plasma electrons. The sheath then takes on a "double sheath" shape (figure 7), which nas a minimum in the potential in front of the probe instead of at the probe. The magnitude of the potential drop in a double sheath is the subject of several theories 9,10 , but simple, analytic solutions are not readily available. While this paper will not go into the derivations of 
the analytic solution of Poisson's equation in the presence of a double sheath, its effects on probe performance will be discussed.

In fact, there are widely differing results from the theories, one of which states that the floating potential of an emitting probe should only be slightly above the floating potential of a non-emitting probe 9 because the emitted electrons from the probe are much colder than the bulk electrons. This derivation has been considered to be quite pessimistic 5 because the derivation can be shown to not always satisfy Poisson's equation in the sheath. In another derivation, the net double sheath drop in the presence of strong secondary emission should be on the order of $k \mathrm{~T}_{e}{ }^{10}$. However, it should be noted that strong secondary electron emission is not expected in PISCES because the electron temperature is not hot enough to have a significantly large population of energetic electrons $(>100 \mathrm{eV})$.

\section{Magnetized Mach Probe Theory}

A mach probe can infer the flow velocity in the bulk plasma from the ratio of the ion saturation currents collectsd by two unidirectional probe tips. Several mach probe theories exist and it is important to discuss how each theory is derived and to what extent such theories are relevant to the experiments being conducted in PISCES. The theories due to P. C. Stangeby 11 and I. H. Hutchinson 12 relate the plasma lensity at the sheath edge of a probe to the bulk plasma flow. However, each theory contains different source terms 
for plasma entering the flux tubes connected to the probe. The Stangeby model relies on uniform cross field diffusion into the flux tubes connected to the probes to balance the collection by the ion saturation probes. The Hutchinson model concerns itself with the effect of plasma viscosity as well as diffusion.

In addition to these two models, Harbour and Proudfoot have developed a semi-empirical model for a mach probe on the DITE tokamak13. The equation for the mach number that Harbour and Proudfoot derive is an approximation to the solutions of the particle and fluid models.

To derive the local mach number from the flex measurements of a pair of magnetized probes, we start with the Stangeby derivation and the basic equations of conservation of mass and momentum and the Boltzmann relation. In oddition, quasineutrality is assumed up to the sheath edge such that $n=n_{i}=n_{e}$, where $n_{e}$ is the electron density. The Boltzmann relation is given by

$$
\mathrm{n}=\mathrm{n}_{\mathrm{o}} \exp \left(\frac{\mathrm{e \Phi}}{\mathrm{k} \mathrm{T}_{\mathrm{e}}}\right)
$$

where $\Phi$ is the potential in the plasma, and $k T_{c}$ is the electron temperature. The conservation of mass equation is

$$
\nabla \bullet(n \mathbf{v})_{\|}=S_{0}
$$

where $S_{0}$ is a source term in the Stangeby derivation due to uniform cross field diffusion and $v$ is the fluid velocity of the plasma within the presheath. The source term $S_{0}$ ignores the effects of variations in 
the potential and density along the perturbed flux tube versus the unperturbed piasma. The uniformity of $S_{0}$ is an assumption used to make the mathematics tenable. As will be seen later in the Hutchinson treatment, the introduction of a non-uniform loss mechanism precludes an analytic solution to the problem. The conservation of ion momentum for warm ions is expressed as

$$
m n v \cdot \nabla v=e n E-\nabla p_{i}-m\left(v-v_{o}\right) S_{o},
$$

where $v_{0}$ is the drift velocity of the ambient plasma. The parallel mach numbers are defined as the fluid velocities $v$ and $v_{0}$ normalized to the ion acoustic speed $c_{s}$ at the sheath edge, which will be shown later to be related to the ion acuustic speed of the ambient plasma $c_{\mathrm{sa}}$ such that

$$
c_{s}=f\left(\frac{T_{i}}{T_{e}}\right) c_{s a}, c_{s a}=\sqrt{\frac{k\left(\gamma_{i} T_{i}+T_{e}\right)}{M_{i}}} .
$$

Thus,

$$
\begin{aligned}
& M \equiv \frac{v}{c_{s}} \\
& M_{0}{ }^{*} \equiv \frac{v_{0}}{c_{s}} \\
& M_{0} \equiv \frac{v_{0}}{c_{s a}}=\frac{M_{0}{ }^{*}}{f\left(T_{i} / \tau_{e}\right)}
\end{aligned}
$$

Finally, assume $?$ constant ion and electron temperature along the field lines, which is a good approximation due to the large thermal conductivity along field lines. The ions are assumed to be isotherma! 
and warm, so that the pressure gradient $\nabla p_{i}$ can be rewritten as follows

$$
\frac{\nabla p_{i}}{m n}=\frac{\gamma_{j} k_{T}}{m n} \nabla_{n}
$$

Taking the derivative of the Boltzmann relation,

$$
\nabla \mathrm{n}=\frac{\mathrm{ne}}{\mathrm{kT}_{\mathrm{e}}} \nabla \Phi=-\frac{\mathrm{ne}}{\mathrm{kT}_{\mathrm{e}}} \mathrm{E}
$$

which gives

$$
\text { en } E=-k T_{e} \nabla \pi
$$

Thus, substituting into (5) yields

$$
\frac{\nabla p_{i}}{m n}-\frac{E}{m}=\frac{k\left(\gamma_{i} T_{i}+T_{e}\right) \nabla n}{m n}=\frac{c_{s}^{2} \nabla_{n}}{n} \text {. }
$$

So, by using (3) and (4), the momentum conservation equation is rewritten in a normalized form

$$
M \frac{d M}{d x}=-\frac{1}{n}\left(\frac{d n}{d x}\right)-\frac{\left(M-M_{0}^{*}\right) S_{0}}{n c_{s}} .
$$

By replacing the fluid velocity in the mass conservation equation (2), the density gradient can be solved for in terms of the mach number

$$
\frac{\mathrm{dn}}{\mathrm{dx}}=\frac{1}{\mathrm{M}}\left(\frac{\mathrm{S}_{\mathrm{o}}}{\mathrm{c}_{\mathrm{s}}}-\mathrm{n} \frac{\mathrm{d} \mathrm{M}}{\mathrm{dx}}\right)
$$

so that equation 8 becomes

$$
M \frac{d M}{d x}=\frac{1}{n}\left(\frac{S_{0}}{M c_{s}}-\frac{n}{M}\left(\frac{d M}{d x}\right)+\frac{\left(M-M_{0}^{*}\right) S_{0}}{c_{s}}\right) .
$$


Solving for $\frac{d M}{d x}$ in (10) gives

$$
\frac{d M}{d x}=\frac{S_{Q}}{n c_{s}}\left(\frac{1+M^{2}-M_{0}^{*}}{1-M^{2}}\right)
$$

Using (11), the constant $\frac{S_{0}}{c_{s}}$ can be replaced in (9), such that

$$
\frac{\mathrm{d} n}{\mathrm{dx}}=\frac{\mathrm{n}}{\mathrm{M}}\left(\frac{\mathrm{dM}}{\mathrm{dx}}\right)\left(\frac{1-\mathrm{M}^{2}}{1+\mathrm{M}^{2}-\mathrm{MM}_{0}^{*}}-1\right) \text {, }
$$

which again can be rewritten to give

$$
\frac{d n}{n}=-d M \frac{2 M-M_{0}^{*}}{1+M^{2}-M_{0}^{*}}
$$

and this can be integrated to give

$$
\ln \left(\frac{\mathrm{n}}{\mathrm{n}_{0}}\right)=-\ln \left(1+\mathrm{M}^{2}-\mathrm{MM}_{0}{ }^{*}\right)
$$

Finally,

$$
\frac{\mathrm{n}}{\mathrm{n}_{\mathrm{o}}}=\frac{1}{1+\mathrm{M}^{2}-\mathrm{MM}_{\mathrm{o}}^{*}}
$$

where $n_{0}$ is the undisturbed density of the plasma outside of the presheath and flux tube. This gives the relationship between the densities and flow velocities in the flux tube and ambient plasma. Plugging (13) back into (11) results in an expression for the mach number gradient in terms of the mach number itself

$$
\frac{d M}{d x}=\frac{S_{0}}{n_{0} c_{s}}\left(\frac{\left(1+M^{2}-M M_{0}^{*}\right)^{2}}{1-M^{2}}\right)
$$

and integrating this equation will give the spatial dependence of $M$ 


$$
\frac{S_{0} x}{n_{0} c_{s}}=\frac{M}{1+M^{2}-M_{0}^{*}} .
$$

From this, the disturbance lengths of the probe can be calculated. The disturbance length determines the minimum distance in front of a surface over which mach number data can be collected accurately. If the wall is within the disturbance length of the probe, then the cross field diffusion will not be able to fill in the flux tube as fast as the probe is depleting it. Thus, the current drawn by the probe will be lower than expected, resulting in an error in the inferred parallel mach number.

To compute the local mach number, as well as the disturbance lengths, some useful boundary conditions have to be applied to (13) and (15). At the sheath of the upstream probe, the Bohm sheath criterion implies that the plasma must be moving at the ion acoustic speed, so that the mach number $M \equiv+1$. Far upstream at the end of the presheath, $M=M_{0}^{*}$ at a position designated as $-x_{v}$ (the origin of our coordinate system being at the probe). In the same way, at the sheath edge of the downstream probe, $M \equiv-1$, and $M=M_{0}^{*}$ at the end of presheath at the position $x_{d}$. Utilizing these boundary conditions, $x_{u}$ and $x_{d}$ can be obtained from (15)

$$
\begin{aligned}
& x_{u}=\frac{n_{0} c_{S}}{S_{0}}\left(-M_{0}^{*}+\frac{1}{2-M_{0}^{*}}\right) \\
& x_{d}=\frac{n_{0} c_{S}}{S_{0}}\left(M_{0}^{*}+\frac{1}{2+M_{0}^{*}}\right)
\end{aligned}
$$

These are the disturbance lengths of the probes in the plasma. 
To compute these quantities, the source term $S_{o}$ and the function $f\left(T_{j} / T_{e}\right)$ has to be known. From (2), this source term can be approximated by cross field diffusion if ionization within the flux tube is neglected. Thus, along the flux tube intercepted by the probe, the cross field diffusion will be related to the source term by

$$
\begin{array}{ll} 
& \Gamma_{\perp} A \cong S_{0} V \\
& D_{\perp} \nabla n x_{i} d \cong S_{0} x_{i} d^{2} \\
& S_{0} \cong \frac{D_{\perp} n_{0}}{d^{2}}
\end{array}
$$

where $\Gamma_{\perp}$ is the flux due to cross field diffusion, $D_{\perp}$ is the cross field diffusion coefficient, $d$ is the size of probe tip, $A$ is the surface area of the fiux tube, and $\mathrm{V}$ is its volume. The source and sink of plasma in the flux tube is shown schematically in figure 8. Plugging (18) into (16), gives finally

$$
\begin{aligned}
& x_{1}=\frac{c_{s} d^{2}}{D_{\perp}}\left(-M_{0}^{*}+\frac{1}{2-M_{0}{ }^{*}}\right) \\
& x_{d}=\frac{c_{s} d^{2}}{D_{\perp}}\left(M_{0}^{*}+\frac{1}{2+M_{0}^{*}}\right)
\end{aligned}
$$

An expression for $f\left(T_{i} / T_{e}\right)$ can be derived by solving for the enhancement of the parallel ion flux at the sheath edge due to the presheath electric field. In addition, the self-consistent change in the field due to the enhanced flux through the sheath has to be taken into account ${ }^{14}$. The enhancement of the ion flux is given by Emmert, et. $\mathrm{al}^{15}$ and can be written 


$$
\Gamma_{\mathrm{i}}=\frac{1}{2 \mathrm{n}_{0}} \mathrm{c}_{\mathrm{s}}=\frac{1}{2 \mathrm{n}_{\mathrm{o}} c_{\mathrm{sa}}} \mathrm{f}(\tau)
$$

where

$$
f(\tau)=\frac{8}{\pi \sqrt{2}}\left(1+\frac{\tau}{Z}\right)^{\cdot 5} \exp (-\Psi) D[\sqrt{\Psi}], \tau=\frac{T_{i}}{T_{c}}
$$

and

$$
D(x)=\int_{0}^{x} t^{2} d t
$$

is the Dawson Integral. $\Psi$ is the presheath potential drop, $\Psi=\frac{\mathrm{e} \Phi}{\mathrm{kT}_{\mathrm{e}}}$. The equation for $\Psi$ at the sheath edge is a result of the potential distribution in the presheath satisfying the Emmert plasma-sheath equation. The expression for $\Psi$ is given as

$$
1=\sqrt{\frac{4 \tau}{\pi Z}} \exp \left(-(1+(Z / \tau) \Psi) D\left[\sqrt{\Psi]}+\operatorname{erf}\left(\sqrt{\frac{Z \Psi}{\tau}}\right)\right.\right.
$$

In the limit of $\tau \ll<1$, which is true for the PISCES plasma, the potential can be approximated by the fluid model solution, $\Psi=\ln 2$, and equation (20) gives 16

$$
f(\tau) \cong \frac{8}{\pi \sqrt{2}}\left(1+\frac{\tau}{2 Z}\right)(.535) \cong .96\left(1+\frac{\tau}{2 Z}\right)
$$

Thus, for the PISCES plasma, where typically $\tau \cong .05$, the difference between the sheath and ambient ion acoustic speed is less than $3 \%$ of the ambient speed. Note that in the case of cold ions, $f(0)=.96$, so that the kinetic and fluid treatments do not exactly coincide in this approximation. However, the value of $\Psi$ used in the kinetic 
treatment comes from fluid theory rather than solving equation (21) in the limit of $\tau$ approaching 0 . From this analysis, for $\tau \ll<1, c_{s} \equiv c_{s a}$ and $\mathbf{M}_{\mathrm{o}}{ }^{*} \equiv \mathrm{M}_{\mathbf{o}}$. On the other hand, in a tokamak application, where $\tau$ is of order unity or greater, this analysis breaks down and equations (20) and (21) must be solved to compute the correct value of $f(\tau)$.

Wili the boundary conditions $M=1$ upstream, $M=-1$ downstream, and $M_{0}{ }^{*} \cong M_{0}$, the local mach number $M_{0}$ can $b$ : computed from the ratios of the upstream and downstream flux measurements. Using the Bohm sheath criterion again, the fluid velocity at the sheath edge of the two probes is found to be equal to the local ion acoustic speed, so that the ratio of the ion fluxes to the two probes is equal to ratio of the densities at the sheath edge, which can be had from (13). Thus,

$$
\frac{\Gamma_{u}}{\Gamma_{d}}=\frac{n(M=+1)}{n(M=-1)}=\frac{1+1+M_{0}^{*}}{1+1-M_{0}^{*}}=\frac{2+M_{0}^{*}}{2-M_{0}^{*}} \equiv \frac{2+M_{0}}{2-M_{0}} \text {. }
$$

Therefore, solving for $\mathbf{M}_{0}$,

$$
M_{0}=2 \frac{\frac{\Gamma_{u}}{\Gamma_{d}}-1}{\frac{\Gamma_{u}}{\Gamma_{d}}+1}
$$

This is the bulk plasma mach number as comuuted by the Stangeby model.

On the other hand, the Hutchinson derivation defines the source of plasma in the flux tube to be due to a true diffusive exchange of ions between the bulk plasma region and the flux tube. 
This varies from the Stangeby model, which considered a source term that is approximately equal to the diffusion rate into an empty flux tube. Diffusion of plasma back sut of the flux tube is not allowed in that mode!. The electrons still obey the Boltzmann relation in the presheath and ambipolar diffusion is still assumed. The source term balances the loss of flux at the probe (equation 2), but the sche term is computed based on the assumption that the ions in the plasma have a mean rate $\Omega$ for diffusive exchange everits, such that

$$
\nabla \bullet(\mathbf{n v})_{\|}=S_{,}=\Omega\left(n_{0}-n\right)
$$

In addition, the effect of the acceleration of the ions in the presheath of the probe must be taken into accosnt in the momentum balance equation. The momentum balance equation (3) then becomes

$$
m n v \cdot \nabla v+m v \Omega\left(n_{0}-n\right)=e n E-\nabla_{p i}-m \Omega\left(n_{0} v_{0}-n v\right)
$$

By applying the Boltzmann relation to (26), the Hutchinson analog to (8) can be derived (assuming the conditions in PISCES of $\tau<<1$ and $\left.\mathbf{M}_{\mathrm{o}}^{*} \equiv \mathbf{M}_{\mathbf{o}}\right)$ :

$$
n M \frac{d M}{d x}=-\frac{d n}{d x}+\Omega \frac{n_{0}}{c_{s}}\left(M_{0}-M\right)
$$

In addition to this normalized expression for the momentum conservation equation, the normalized mass conservation equation can be rewritten 


$$
M \frac{d n}{d x}+n \frac{d M}{d x}=\frac{\Omega}{c_{s}}\left(n_{0}-n\right) .
$$

Equations for $\frac{d n}{d x}$ and $\frac{d M}{d x}$ can be found from plugging (27) into (28), which gives

$$
\frac{d M}{d x}=\frac{\Omega}{c_{s}}\left(\frac{n_{0}\left(1-M_{0}+M^{2}\right)-n}{n\left(1-M^{2}\right)}\right)
$$

and

$$
\frac{d n}{d x}=\frac{\Omega}{c_{s}}\left(\frac{n_{0}\left(M_{0}-2 M\right)+n M}{1-M^{2}}\right) .
$$

Finally, by dividing ( 30 ) by (29), we get the equation

$$
\frac{d n}{d M}=n \frac{n_{0}\left(M_{0}-2 M\right)+n M}{n_{0}\left(1-M_{0}+M^{2}\right)-n}
$$

which has the boundary condition $\frac{d n}{d M}(0)=n_{0}$.

This is a highly nonlinear differential equation, that can only be solved by gross approximation or numerical methods. Once again, the sheath criterion is applied at the probe sheath $(M=1$ for the upstream probe, and $M=-1$ for the downstream probe). While the numerical approximation method is too cumbersome to be quoted here, by approximating $n$ in a second order Taylor series expansion about $M_{0}$, the solution to (31) will be of the form

$$
n\left(M, M_{0}\right)=p^{\alpha} e^{-q}
$$


where $\alpha, p$, and $q$ are polynomials of $M$ and $M_{0}$. The solution for $M_{0}$ in terms of $\Gamma_{\mathrm{u}} / \Gamma_{\mathrm{d}}$ is found by determining the roots to the non-linear equation

$$
\frac{n\left(M=1, M_{0}\right)}{n\left(M=-1, M_{0}\right)}-\frac{\Gamma_{1}}{\Gamma_{d}}=0
$$

such that $M_{0}$ is simply a function of $\frac{\Gamma_{y}}{\Gamma_{d}}$.

The practical upshot of the theory is that the Hutchinson model will predict subsonic ion flow at higher upstream to downstream flux ratios than the Stangeby model. This is due to the different form of the source term and the fact that particles can not only enter the flux tube but can also leave it before being collected, thus providing a momentum sink in the system.

The Harbour and Proudfoot model 13 introduced earlier in this section was also tested for the mach probe data taken with the PISCES fast scanning probe. This model includes the effect of the ion energy distribution on the flow of ions towards the sheath edge. Harbour and Proudfoot basically fold this energy dependence term into the fluid model and return with a simple expression for the mach number in terms of the upstream to downstream flux ratio

$$
\mathrm{M}=0.6 \ln \left(\frac{\Gamma_{\mathrm{u}}}{\Gamma_{\mathrm{d}}}\right)
$$

This expression is a semi-empirical "average" of the ion energy distribution correction and the Stangeby fluid model discussed in 
depth earlier. The difference in the mach numbers computed by these two schemes can vary considerably and can possibly be attributed to the fact that the assumptions made may not hold very well, especially for the downstream probe. In the Stangeby fluid model, the fact that the plasma collected by the downstream probe has to reverse its flow direction to meet the sheath criterion may overestimate the amount of flux collected by the probe for a given ambient mach number. On the other hand, the effect due to the ion energy distribution correction assumes that the probes are sampling an undistributed region of plasma with the same ion energy distribution 17. Harbour and Proudfoot have considered that neither of these assumptions may hold and thus developed their "average" formula.

As a way of comparing these models, each one is plotted versus upstream to downstream flux ratio. As can be seen in figure 9, the Stangeby model predicts the highest mach number for any given flux ratio, while the other models predict subsonic ion flow at higher flux ratios than the Stangeby model, which goes supersonic at $\frac{\Gamma_{y}}{\Gamma_{d}}=3$. It should also be noted here that the Stangeby model also covers supersonic flow, and the solution for the mach number is not covered here but is included in the computation of figure 9 . In the results section of this paper, these various mach probe models will be compared using actual data taken with the PISCES fast scanning probe and conclusions regarding the validity of the various models in PISCES tokamak-like edge plasmas will be discussed. 


\section{Probe Emission Testing}

One of the i:iportant questions that has to be answered about the emissive probe is whether it is actually emitting a sufficient electron flux during its complete scan through the plasma. An experimental verification of this is to observe the variation of the measured potential versus the voltage needed to heat the probe tip. If an emissive probe is operated in strong emission, then its potential is determined by the plasma's potential and temperature and should not be a function of the amount of emission from the probe. On the other hand, if the probe's emission is temperature limited, then the potential dron in the double sheath can also be expected to be a function of temperature. As can be seen in figures 10 and 11 , the emitting probe has a small region of temperature limited emission followed by a larger region of space charge limited emission. The slow variation in the measured space potential once the probe is strongly emitting can possibly be explained by the presence of a hot electron component of the electron velocity distribution. If a nonMaxwellian electron distribution exists in the plasma, then the bulk electrons are more likely to be reflected in the sheath than the hot electron component, so that the hot electrons are a larger component of the net electron current at the probe, and the energy distribution will therefore deviate further from a Maxwellian.

The electrostatic sheath is not the only mechanism that can control the emission from the filament. Since the potential 
measurement is taken with the filament heating current on, there is an oscillating magnetic field around the wire that is perpendicular to the probe's sheath. If the Larmor radius of the emitted electrons in this field is smaller than the sheath thickness, then the electrons will not be accelerated out of the sheath freely. This will limit the total emission current from the probe. However, for typical plasma conditions in PISCES $\left(T_{e}=10 \mathrm{eV}, n_{e}=10^{12} \mathrm{~cm}^{-3}\right)$, the Debye length $\lambda_{D}$ $=.02 \mathrm{~mm}$ while the Larmor radius of the emitted electrons in the magnetic field at the wire surface $(I=4$ Amps, $B=60 G)$ is $r_{L_{e}}=$ $.2 \mathrm{~mm}$. The sheath thickness is approximately $\mathrm{d}=3 \lambda_{\mathrm{D}}$, so that $\mathrm{r}_{\mathrm{Le}} \gg$ d. Thus, the field induced by heating the filament during the measurement does not significantly alter the emission.

Other important considerations in the operation of the fast scanning emissive probe are thermal reliability and maximum shot repetition rate. Since the actual temperature distribution in the probe shaft and the leads can not be measured, a one dimensional heat traisfer calculation was done to estimate the temperature that the filament and braze reach to ensure the longevity of the probe. The concern here was that the braze joint between the tungsten and copper might undergo severe temperature excursions during the heating phase of the probe operation, thereby causing the braze to flow and shorten the life of the probe.

The heat transfer equation is

$$
\rho c_{p} \frac{d T}{d t}=k \frac{d^{2} T}{d x^{2}}+\frac{\left(P_{\text {iquje }}+P_{\text {plasma }}-P_{\text {rad }}\right)}{V}
$$


where

$$
P_{\text {joule }}=I^{2} R, P_{\text {plasma }}=\Gamma_{e} k T_{e} \text {, and } P_{\text {rad }}=\varepsilon \sigma T^{4}
$$

Since the variation in the thermodynamic properties of the materials and the radiative power loss are not negligible in this case the differential equation becomes non-linear and must be solved numerically. The numerical calculation includes the thermal variation of the emissivity, resistivity, and thermal conductivity of the tungsten. The thermal conductivity and heat capacity of the brazing material is ignozed, but the effects of an alumina jacket around the copper leads are included. In order to simulate the contact between the copper conductor and the alumina, heat is allowed to conduct from the conductor to the alumina at a fraction of the alumina's conductivity. In the computations shown here, the conductivity to the alumina is grossly approximated by $10 \%$ of alumina's true thermal conductivity.

The results of these calculations show that the alumina plays a critical role for the probe's reliability because it acts as a heat sink ip the braze and leads. Without the alumina jacket in the model, the braze and copper leads see a severe thermal excursion (figure 12). This thermal excursion approaches an arbitrary maximum temperature of $1000^{\circ} \mathrm{C}$ for the braze joint, so it is likely that the braze will lose its mechanical strength after repeated heatings. On the other hand, if the alumina jacket is present and heat is allowed to pass from the copper and braze into the alumina, then the severe 
thermal excursion is not observed, as seen in the second case overplotted in figure 12 .

Figure 13 shows a typical probe equilibrium temperature profile. The equilibrium temperature profile is characterized by a steep temperature gradient in the tungsten near the braze joint, with a least $75 \%$ of the probe tip's length above $2000^{\circ} \mathrm{C}$ (the typical temperature for strong emission in the PISCES plasma). Since a large fraction of the probe area will be in strong emission, the potential measurement should be unaffected by probe geometry effects.

It is important that a large heating current flow through the probe so that the tip will heat rapidly and its resistivity will be greater than the leads. If the probe is heated too cautiously, then the entire probe structure will heat up slowly rather than the tip being heated rapidly. In particular, this could lead to long term thermal cycling stresses on the alumina.

Finally, as for the question of the repetition rate, figure 14 shows the thermal history a few seconds after the heating current is shut off. The tip cools off more rapidly than the rest of the structure due to radiation, which dissipates most of the heat a few seconds after the heating phase. From these results, the probe can be repetitively heated with a period of about 5 seconds without overheating the leads or braze. In typical PISCES operations, this limit is not approached because of the dead time between shots necessary for data acquisition and analysis (typically 1 minute). 
Occasionally, the diagnostic is fired repetitively without the digitizers enabled in order to observe real time density profiles on a storage uscilloscope during periods of discharge tuning. However, the emissive probe is not externally heated when the diagnostic is operated in this fashion, and the heat flux from the plasma is negligible because of the probe's small surface area.

\section{Results from Data Collected by the Probe}

Representative single shot spatial profiles of the floating and space potential in the PISCES plasma column are shown in figure 15. The space potential profile is typical of reflex arc discharges 18 because the electrons diffuse much more slowly across the magnetic field than the ions. The cross field diffusion coefficients of a partially ionized plasma in a strong magnetic field can be shown ${ }^{19}$ to be proportional to the ion and electron temperature and mass ratios

$$
\frac{D_{L_{\varepsilon}} \alpha}{D_{L i}}\left(\frac{T_{e}}{T_{i}}\right)\left(\frac{m_{e}}{m_{i}}\right)
$$

The proportionality of the cross field mobility coefficients can also be shown to be

$$
\frac{\mu_{\perp e}}{\mu_{\perp i}} \alpha \frac{m_{\varepsilon}}{m_{i}}
$$

In PISCES, typically $\frac{m_{e}}{m_{j}} \ll \frac{T_{i}}{T_{e}}$, so that $\frac{D_{\perp e}}{D_{\perp i}}<1$. The radial particle fluxes to the wall are 


$$
\begin{aligned}
& \Gamma_{\mathrm{ri}}=\mu_{\perp \mathrm{i}} \mathrm{n} \nabla \Phi-D_{\perp \mathrm{i}} \nabla n \\
& \Gamma_{\mathrm{re}}=-\mu_{\perp e^{n}} \mathrm{\nabla \Phi}-D_{\perp \mathrm{e}} \nabla n
\end{aligned}
$$

If the parallel losses to the endplates are neglected, then in order to have ambipolar diffusion of the plasma across the field lines, the ion and electron fluxes have to be the same. Since the ions diffuse across the field lines faster than the electrons, a radial electric field (or potential gradient) is formed to electrostatically confine the ions in the plasma. In reality, the depth of thi: electrostatic potential well is determined not only by the cross field diffusion and mobility, but also by the parallel losses to ends of the machine, the presence of a hot emitting cathode, and the difference in ion-neutral and electronneutral collision frequencies, which was also neglected in equations $36 a$ and $36 b$.

Three dimensional profiles are produced by accumulating fast scanning probe shots at different $X-Y$ table positions. Data is taken at regular spatial intervals to simplify the data reduction. Figures 16 through 18 show the space potential, density, and mach number profiles of the presheath in front of a material sample. The presheath is the region in the plasma in front of the sheath and it supports a potential drop which accelerates ions to the sound speed at the sheath edge, as required by the Bohm sheath criterion. The potential drop necessary to accelerate the ions in a plasma is $\Delta \Phi \equiv$ $0.5 \mathrm{kT}_{\mathrm{e}}{ }^{20}$. Plugging this potential variation into the Boltzmann relation shows that the plasma density between the ambient plasma 
and the sheath edge should fall by approximately a factor of two21. The density drop off is due to the fact that while the flux, which is the product of the density and the velocity, is constant along the field lines, the ions are accelerating and therefore, the density has to fall.

The mach number profile is computed by the Stangeby model, and it can be seen in the figure that the mach number actually exceeds unity near the sample surface. This is due to the fact that the sample surface is within the disturbance length of the probe and the mach probe models break down when this occurs. The downstream disturbance length for the probe as computed by (19b) in a $10 \mathrm{eV}$ plasma with a $1 \mathrm{kG}$ magnetic field is approximately $1 \mathrm{~cm}$. Therefore, the mach number data within the last $1 \mathrm{~cm}$ of the wall surface is not entirely reliable.

The various mach probe models summarized earlier in this paper were compared for the same set of data and are shown in figure 19. As can be seen in the figure, the Stangeby model is the only model to predict a sound speed flow near the wall surface. All the other models predict lower values of the mach number near the wall. To compare the various models, a simple particle free fall method has been developed 22 . The free fall model assumes an initial velocity comparable to the mach number far upstream from the material sample, and the ions are allowed to fall through the potential drop in the presheath. The free fall model is described by a simple energy conservation equation 


$$
M(x)=\sqrt{M^{2}(0)+\frac{2(\Phi(0)-\Phi(x))}{k T_{e}}}
$$

It should be noted that this equation does not include any of the parallel momentum loss terms taken into account by the more sophisticated models that are being compared here. Thus, equation (38) is the maximum possible change in the mach number for a given potential drop. By setting the normalization $M(0)$ equal to the Stangeby mach number computed far upstream, the free fall model predicts that the mach number should be near unity near the sheath edge. This gives confidence that the model is somewhat accurate in predicting the dependence of the mach number on the potential. It also suggests that parallel momentum loss mechanisms are not important to the computation of the mach number. If they were, then equation (38) would have predicted $M \gg>1$ at $x=0$. The other models compute lower mach numbers far from the surface, as compared to the Stangeby model. However, the free fall profile is relatively insensitive to these lower initial velocities and continues to predict a mach number near unity at the sheath edge. The Harbour and Proudfoot model and the Hutchinson model predict lower mach numbers throughout the presheath and have trouble achieving the unity mach number condition at the sheath edge.

The density profile is computed with an average of the flux collected by the upstream and downstream probes using the formula

$$
<\mathrm{n}(\mathrm{x})\rangle=\frac{\Gamma_{\mathrm{u}}+\Gamma_{\mathrm{d}}}{4 \mathrm{c}_{\mathrm{s}}}
$$


The density profile along a particular flux tube can be compared to a density computed from the space potential via the Boltzmann Relation

$$
n(x)=n_{0} \exp \left(\frac{\operatorname{e\Phi }_{s}(x)}{k T_{c}}\right)
$$

The comparison between (39) and (40) and several other representative profiles is shown in figure 20 . Normalizing the density derived from the Boltzmann Relation to the measured average density far upstream from the surface produces the best fit between the two data sets.

The three dimensional profiles of potential, density, and mach number that have been shown here have good correlation between theory and experiment in many respects, but there is one very important disagreement. The relationship between the floating and space potential measured from the $1-V$ characteristic of a cold probe in a helium plasma is 8

$$
\Phi_{s}-\Phi_{f}=3.5 k T_{e} / e
$$

However, the difference $\Phi_{S}-\Phi_{f}$ as measured with a hot and cold filament, respectively, is only about $2.0 \mathrm{kT} e$ near the center of the discharge. This could be possibly attributed to the stray capacitance in the cable from the probe to the data acquisition electronics. The stray cable capacitance forms a non-linear, low impedance path to ground for fluctuations in the potential, and coupled with the 
plasma's non-linear response to fluctuations, oscillations about the floating potential do not average out to zero.

In order to analyze the effect of fluctuations on the time averaged floating potential, a few basic relations are necessary. In a plasma whose particles are maxwellian, the space and floating potentials are related by

$$
V_{s}-V_{f}=\alpha k T_{e} / e
$$

where $\alpha$ is some constari. The current drawn by a probe in the plasma at a voltage $V_{p}$ near the floating potential is given by 23

$$
I_{n e t}=I_{e} \exp \left(-\left(\frac{e\left(V_{s}-V_{n}\right.}{k T_{e}}\right)\right)-I_{\text {sat }}
$$

Note here that the electron currents are positive. If the probe electronics have an input impedance $Z$, then the probe voltage will be

$$
V_{p}=-I_{n e t} Z
$$

The I-V characteristics described by equations 43 and 44 are shown in figure 21.

Using equation 42 , the equation for the net current from the probe (43) becomes

$$
I_{n e t}=I_{e} \exp \left(-\left(\frac{e\left(V_{f}+\left(\alpha k T_{e} / e\right)-V_{p}\right)}{k T_{e}}\right)\right)-I_{s a t}
$$

$I_{e}$ and $I_{s a t}$ are relat $\perp$ by the true floating potential condition $I_{\text {net }}=0$ when $V_{p}=V_{f}$, thus 


$$
I_{e} \exp (-\alpha)=I_{\text {sat }}
$$

and therefore

$$
I_{\text {net }}=I_{\text {sat }}\left(\exp \left(-\left(\frac{e\left(V_{f}-V_{0}\right)}{k T_{e}}\right)\right)-1\right)
$$

A floating probe and its electronics can be described by the simple network snown in figure 22 . The resistance $R_{s}$ is the source impedance of the plasma while the resistance $R_{T}$ is the input impedance of the nueasurement circuit. The capacitor is due to the intervening length of cable between the probe and electronics. If fluctuations are introduced into this derivation, the following substitutions can be made

$$
\begin{aligned}
& V_{f}=V_{f o}+\widetilde{v}_{f} e^{i \omega t} \\
& V_{p}=V_{p o}+\widetilde{v}_{p} e^{i \omega t} .
\end{aligned}
$$

Then, an analysis of the network in figure 22 can be made, such that

$$
\tilde{v}_{p}=\frac{\tilde{v}_{f}}{\sqrt{\left(1+\left(R_{s} / R_{T}\right)\right)^{2}+\left(R_{s} C \omega\right)^{2}}}
$$

For ease of notation, the following definition is made

$$
\delta=\sqrt{\left(1+\left(R_{S} / R_{T}\right)\right)^{2}+\left(R_{s} C \omega\right)^{2}} .
$$

Applying equations $48 \mathrm{a}$ and $48 \mathrm{~b}$ to equations 44 and 47 gives

$$
-\left(V_{p o}+\widetilde{v}_{p} e^{j \omega t}\right)=Z I_{s a t}\left(\exp \left(-\left(\frac{e\left(V_{f o}+\widetilde{v}_{f} e^{i \omega t}-V_{p o}-\widetilde{v}_{p} e^{i \omega t}\right)}{k T_{e}}\right)\right)-1\right)
$$


anc. using (49) and time averaging over many periods, such that

$$
\int_{T}^{j} f^{i \omega t} d t=0 \text { and } \int_{T} \exp \left(e^{i \omega t}\right) d t=1
$$

gives

$$
-V_{p o}=Z_{s a t}\left(\exp \left(-\left(\frac{e\left(V_{f o}-V_{p 0}+\nabla_{f}(1-1 / \delta)\right)}{k T_{e}}\right)\right)-1\right)
$$

Multiplying by $\frac{\mathrm{e}}{\mathrm{kT}_{\mathrm{c}}}$ gives

$$
-\frac{e V_{p O}}{k T_{e}}=\frac{e Z I_{S a L}}{k T_{e}}\left(\exp \left(-\left(\frac{e\left(V_{f o}-V_{p o}+\widetilde{v}_{f}(1-1 / \delta)\right)}{k T_{e}}\right)\right)-1\right)
$$

By defining

$$
\begin{array}{ll} 
& \Psi_{\mathrm{p}}=\frac{e V_{\mathrm{po}}}{k T_{e}}, \Psi_{\mathrm{f}}=\frac{\mathrm{eV} \mathrm{V}_{\mathrm{fo}}}{k T_{e}}, \widetilde{\Psi}_{\mathrm{f}}=\frac{e \widetilde{\nabla}_{\mathrm{f}}}{k T_{e}}, \\
\text { and } \quad & y=\frac{e Z I_{\text {sat }}}{k T_{e}} .
\end{array}
$$

then (53) becomes

$$
-\Psi_{p}=\gamma\left(\exp \left(-\left(\Psi_{f}-\Psi_{p}\right)+\tilde{\Psi}_{f}(1-1 / \delta)\right)-1\right)
$$

By noting that the source impedance of the plasma near $V_{f}$ is 24

$$
R_{s}=\frac{k T_{e}}{\mathrm{eI}_{\text {sat }}} \text {, }
$$

then $\gamma=Z / R_{5}$ is the ratio of the input to source impedance of the diagnostic. 
Finally, by rearranging (54), an expression for the floating potential in terms of the probe's potential can be derived

$$
\Psi_{f}=\Psi_{p}-\Psi_{f}(1-1 / \delta)-\ln \left(1-\Psi_{p} / \gamma\right)
$$

The size of the error due to the non-linear response of the diagnostic system can be expressed as

$$
\Psi_{p}-\Psi_{f}=\widetilde{\Psi}_{f}(1-1 / \delta)+\ln \left(1-\Psi_{p} / \gamma\right)
$$

To evaluate the size of the error due to this effect, typical values can be inserted:

$$
\begin{array}{ll}
\mathbf{R}_{\mathrm{T}}=10^{6} \Omega & \mathbf{k T}_{\mathrm{e}}=15 \mathrm{eV} \\
\mathrm{C}=10^{-9} \mathrm{~F} & \mathrm{I}_{\text {sat }}=7.5 \times 10^{-4} \mathrm{~A} \\
\mathbf{R}_{\mathrm{s}}=2 \times 10^{4} \Omega &
\end{array}
$$

and $\delta$ and $\gamma$ are computed for an average frequency response of $10 \mathrm{kHz}$ (the typical oscillation frequency in PISCES) and taking $\widetilde{\Psi}_{\mathrm{f}} \equiv$ 325. Therefore, for $\gamma=.8, \delta=2.6$, and $\Psi_{p}=-3$, then $\Psi_{p}-\Psi_{f}=1.7$. This can easily account for the experimental result $\Phi_{\mathrm{s}}-\Phi_{f}=2.0 \mathrm{kT} / \mathrm{e}$ since the floating probe potential is much greater than the true floating potential. If, on the other hand, the cable capacitance is reduced to $100 \mathrm{pF}$ by shortening the cable to one meter, then the value of $\Psi_{p}-\Psi_{f}$ becomes .3 , which is more acceptable.

However, the effect of stray cable capacitance on the time averaged floating potential measurement has not been observed in PISCES so far. The fast probe diagnostic intended for studying plasma profiles in CCT was mounted on PISCES and floating potential 
data from it was compared to the floating potential data from the PISCES fast probe. The data acquisition electronics for the CCT fast probe have been designed to minimize stray cable capacitance, which is estimated to be no greater than $200 \mathrm{pF}$. A comparison of floating potential measurements from the two diagnostics actually coincide within 5 volts, which is much less than the $\mathrm{kT}_{e}$ that would be expected from the analysis.

While the derivation above can not necessarily be extended to space potential measurements, the fact that the probe is capable of emitting electrons will decrease the source impedance of the plasma to about $1 \mathrm{k} \Omega$. Thus, the effect of oscillations on the space potential measurement should be much less severe than in the floating potential.

There may be an additional discrepancy in the difference $\Phi_{s}$ $\Phi_{f}$ due to the existence of a double sheath in front of the emissive probe. The depth of the double sheath is unfortunately masked in the uncertainty of the floating potential measurement. However, there is still some evidence that the sheath fo: mation around the emitting probe affects the space potential measurement. The emissive probe I-V characteristic was collected and compared to the I-V characteristic in its non-emitting state. Theoretically, when the probe potential is above the true space potential, the emitted electrons will see a potential hill that is too high to climb since they are emitted from the wire with a temperature of leV. The collected electron current will then simply depend upon the random electron 
current from the plasma. Thus, the I-V characteristics should coincide above the space potential. Below the true space potential, the electrons emitted from the probe are no longer trapped by the sheath potential and they can enter the plasma. The emitted electron current entering the plasma will decrease the net electron current collected by the probe. Thus, the non-emitting and emitting I-V characteristics will begin to diverge. However, this explanation breaks down in this experiment because of the coupling of the sheath impedance to the cable capacitance.

In order to collect the I-V characteristics, the fast probe was fixed at a particular position on the $X-Y$ table such that the probe passed through the center of the discharge. Thus, the cylindrical symmetry was preserved. The emissive probe filament was then biased at a fixed voltage and the current profile collected by the probe was recorded for the emitting and non-emitting cases. The I$\mathrm{V}$ characteristic was then reconstructed by taking many fast probe shots covering a range of voltages from below the floating potential to slightly above the space potential. For comparison, the temperature at various radial positions was taken with the PISCES water cooled fixed Langmuir probe.

Figure 23 shows the two characteristics at the centerline of the plasma column, and it is clear that the floating potential of the emitting probe is about $1.5 \mathrm{kT}_{e}$ below the divergence point of the two characteristics. Again, this corresponds to the observed discrepancy in the numerical constant of equation 41 . 
These measurements highligit the advantages and limitations of this form of probe measurement. In regions of constant electron temperature and fluctuations, such as along field lines, the error in the space and floating potential measurements should be constant. Therefore, while the probe may not measure the absolute value of the potentials, it will still measure the variation in the potentials very accurately. In fact, in the presheath experiments done in PISCES, it is unnecessary to know the absolute value of the space potential in order to compare the variation of the density and space potential via the Boltzmann relation.

The difficulty with this diagnostic arises when the probe is used to compare potential variations across electron temperature gradients and in regions with difforent levels of fluctuations. It is currently unclear if the difference between the probe potential and the true space potential becomes a function of space due to the electron temperature profile. Once the capacitive effects of the measuring technique are minimized, then a more detailed study of the effect of the sheath around the emissive probe can be made. If the sheath effects are significant, then quantities such as the radial electric field, which is important to the overall confinement of the ions in PISCES and, more specifically, to biased limiter simulation experiments done in PISCES 26 , can be over-estimated because the depth of the potential well measured with the floating emitting probe is too great. 


\section{Future Diagnostic Development}

Obviously, the capacitive effects of the diagnostic have to be minimized in order to measure the floating potential properly. This is easily accomplished by reducing the length of cable between the diagnostic and the electronics. If this is insufficient, then there are advanced methods for actively driving the shield of the cable to reduce the impedance to ground.

in order to avoid any adverse effects due to temperature gradients on the space potential measurement (which is very important if this diagnostic were to be employed as a tokamak edge plasma monitor), a more sophisticated probe would have to be constructed. Since the true space potential is characterized by being at the divergence point of the emitting and non-emitting $I-V$ characteristics, a feedback circuit can then be used to fix the bias on an emitting and non-emitting probe to the minimum potential at which each probe collects the same amount of current. Tnis is known as a differential emissive probe 27 because the diagnostic depends upon a high speed, high voltage differential amplifier to drive the probe voltage (figure 24). It is expected that the error in the potential measurement will be smaller because the diagnostic no longer depends on the sheath physics occurring in front of the probe. The main sources of error can be expected to come from the thermal energy of the emitted electrons and any secondary electron emission from hot electron bombardment. The secondary electron emission problem can be minimized by heating the non-emitting probe to boil 
off any impurities which form on the filament. Then, both probes will have to same secondary electron emission coefficient, and if the hot electron population on each probe is similar, then the secondary electron emission will be the same for each probe and will not be detected by the differential amplifier circuit.

The scanning differential emissive probe is not a trivial extrapolation of the current diagnostic being operated. The two emissive probe loops must have the same dimensions within a very small percent, otherwise the difference in the collection areas of each probe will be too large to correct for in the sensing electronics. The other difficulty in this design is in the differential amplifier circuit. Depending upon the voltage slew rate required by the size of the potential gradients encountered and the velocity the probe through the gradients, the differential amplifier circuit may operate near the stability limit of its high voltage and current components.

\section{Conclusions}

A fast spatially scanning diagnostic for producing two dimensional profiles of floating and space potential, plasma density, and parallel mach number has been developed. The generic design of the pneumatic actuator is adaptable to tokamaks, as can be seen by the use of probes with designs similar to PISCES fast probes on the TEXTOR and CCT machines, but the development of this diagnostic on PISCES allows for the testing of scanning emissive probe designs 
in a simpler machine. The direct measurement of the space potential by the means of a floating, emitting filament may be complicated by the presence of a double sheath, but this can be corrected for by construction of a scanning differential emissive probe. The floating potential measurement may been affected by significant stray capacitance and future experiments will attempt to observe and correct the effect. The fast scanning probe diagnostic has observed the structure of the electrostatic presheath in front of a wall surface perpendicular to the applied magnetic field and will be used to observe the effect on the presheath of the magnetic sheath in front of a wall surface at an oblique angle to the magnetic field 28 . In addition, this diagnostic is being used to measure potential and density profile modification in the presence of biased structures in the plasma, but the calculation of radial electric fields from the space potential data may be hampered by the effect of radial temperature gradients on the probe potential. The application of a scanning differential emissive probe should also minimize the error induced the space potential measurement by temperature gradients. Finally, the diagnostic is weing used to monitor plasma potential and density profiles in several other experiments, including ongoing experiments on radiation-enhanced sublimation of graphite 29 and biased pumped divertor simulation experiments ${ }^{30}$. 


\section{Acknowledgements}

I would like to thank Dr. Lothar Schmitz for many hours of stimulating discussions and constructive criticism and Dr. En Yao Wang, from the South Wesiem Institute of Physics, Leshan, China, for information on the differential emissive probe. The talents of Tom 'jketchley, John Elverum, Greg Gunner, and Kirk Andrews are also appreicated in keeping the PISCES facility in working order. 


\section{Bibliography}

1. B. LaBombard, R. W. Conn, R. Lehmer, W. K. Leung, Effect of Applied Electrical Bias on Edge Plasma Profiles and Plasma Elows in PISCES SOL Simulation Experiments, Bull. Amer. Phys. Soc. 32 (1987) 1941

2. N. Hershkowitz, M. H. Cho, Measurement of Plasma Potential Using Collecting and Emittine Probes, J. Vac. Sci. Technol. A $\underline{6}$ (1988) 2054-2059

3. N. Hershkowitz, B. Nelson, J. Pew, D. Gates, Self-Emissive Probes, Rev. Sci, Instrum. 54 (1983) 29-34

4, E, Y, Wang, T. Intrator, N. Hershkowitz, Direct Indication Technique of Plasma Potential with Differential Emissive Probe, Rev. Sci. Instrum. 56 (1985) 519-524

5. E. Y. Wang, N. Hershkowitz, D. Diebold, T. Intrator, R. Majeski, H. Persing, G. Severn, B. Nelson, Y. J. Wen, Secondary Electron Emission-Capacitive Probes for Plasma Potential Measurements in Plasmas with Hot Electrons, J. Appl. Phys. 61 (1987) 47864790

6. D. M. Goebel, G. A. Campbell, R. W. Conn, Plasma Surface Interaction Experimental Facility (PISCES) for Material and Edge Physics Studies, J. Nucl. Mater. 121 (1984) 277-283

7. K. S. Chung, I. H. Hutchinson, B. Lipschultz, Plasma Flow Measurements Along the Presheath of a Magnetized Plasma, Bull. Amer. Phys. Soc 33 (1988) 2103

8. P. C. Stangeby, "The Plasma Sheath," in D. E. Post and R. Behrisch, Physics of Plasma-Wall Interactions in Controlled Fusion, Plenum (1986) 41.97

9. F. F. Chen, Double Sheaths, Chapter 7 of a PPPL report of 1962

10. V. L. Sizonenko, Effects of Sirong Secondary Electron Emission on a Plasma Column, Sov. Phys. Tech. Phys. 26 (1981) 1345-1350 
11. P. C. Stangeby, Measuring Plasma Drift Velocities in Tokamak Edge Plasmas Using Probes, Phys. Fluids 27 (1984) 2699-2704

12. I. H. Hutchinson, A Fluid Theory of Ion Collection by Probes in Strong Magnetic Fields with Plasma Flow, Phys. Fluids 30 (1987) $\quad 3777-3781$

13. G. Proudfoot, P. J. Harbour, J. Allen, A. Lewis, Poloidal and Radial Variations in Plasma Transport in a Limiter Scrape-Off Layer in DITE, J. Nuc. Mat. 128-129 (1984) 180-185

14. P. C. Stangeby, Plasma Sheath Transmission Factors for Tokamak Edge Plasmas, Phys. Fluids 27 (1984) 682-690

15. G. A. Emmert, R. M. Wieland, A. T. Mense, J. N. Davidson, Electric Sheath and Presheath in a Collisionless. Finite Ion Temperature Plasma, Phys. Fluids 23 (1980) 803-812

16. P. C. Stangeby, Effect of Bias on Trapping Probes and Bolometers for Tokamak Edge Diagnosis, J. Phys. D 15 (1982) 1007-1029

17. P. J. Harbour, G. Proudfoot, Mach Number Deduced from Probe Measurements in the Divertor and Boundary Laver of DITE, J. Nuc. Mat. 121 (1984) 222-228

18. F, F, Chen, Badial Electric Field in a Reflex Discharge, Phys. Rev. Let. 8 (1962) 234-237

19. F. F. Chen, Introduction to Plasma Physics and Controlled Eusion.Vol. 1, Plenum 2nd. ed. (1984) Ch. 5

20. see ref. 7

21. F. F. Chen, Introduction to Plasma Physics and Controlled Fusion.Vol. 1, Plenum 2nd. ed. (1984) Ch. 8

22. B. LaBombard, R. W. Conn, Y. Hirooka, R. Lehmer, W. K. Leung, R. E. Nygren, Y. Ra, G. Tynan, Presheath Profiles in Simulated Tokamak_Edge Plasmas, UCLA-PPG-1I47 (1988)

23. F. F. Chen, "Electric Probes," Chapter 4 in R. H. Huddlestone and S. L. Leonard, Plasma Diagnostic Techniques, Academic (1965) 
24. S. Yoshikawa, "Low Frequency Instabilities," Ch. 8 in H. R. Griem and R. H. Lovberg, Plasma Physics. Part A, Academic (1970)

25. G. Tynan, private communication.

26. B. LaBombard, R. Lehmer, K. S. Chung, R. W. Conn, Y. Hirooka, R. E. Nygren, Biased SOL Simulation Experiments in PISCES: An Investigation of the Use of Electrical Bias to Modify Tokamak SOL Transport, Bull. Amer. Phys. Soc 33 (1988) 2103

27. see ref. 4

28. R. Chodura, "Plasma Flow in the Sheath and the Presheath of a Scrape-Off Layer," in D. E. Post and R. Behrisch, Physics of Plasma-Wall Interactions in Controlled Fusion, Plenum (1986) $99-134$

29. R. E. Nygren, A. Pospieszczyk, R. W. Conn, D. Goebel, Y. Hirooka, B. LaBombard, R. Lehmer, Y. Ra, Plasma Erosion of Graphite at High Temperature, Bull. Amer. Phys. Soc 33 (1988) 2103

30. PISCES Program: Summary of Research 1988, UCLA-PPG-1 187 (1988) 


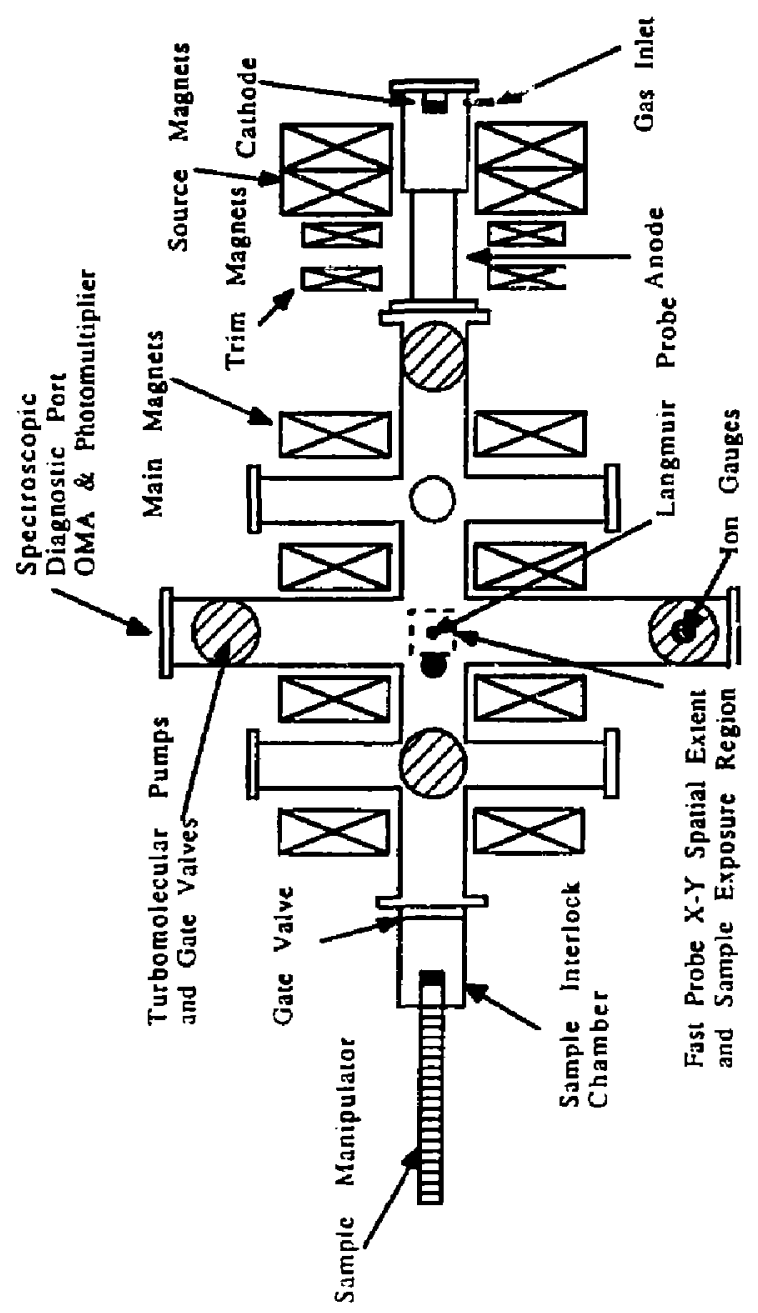

Figure 1 - General schematic layout of the PISCES experimental facility, showing the fast probe diagnostic position in relation to the plasma generator, magnetic field, and other diagnostics. 


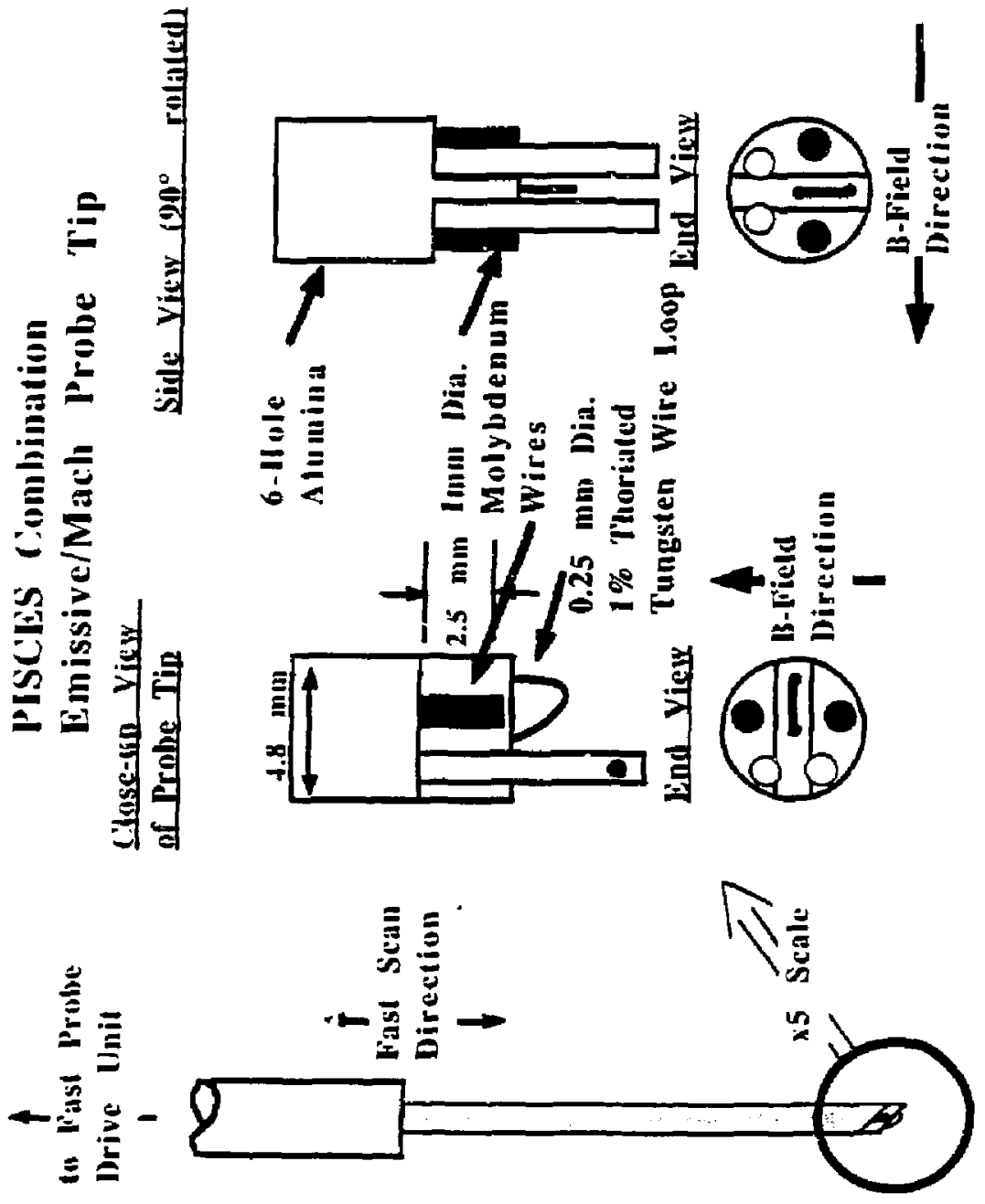

Figure 2 - Detailed schematic diagram of the scanning combination emissive and mach probe tip showing dimensions and relative position of the probes. 


\section{PISCES Fast-Scanning Probe Drive}

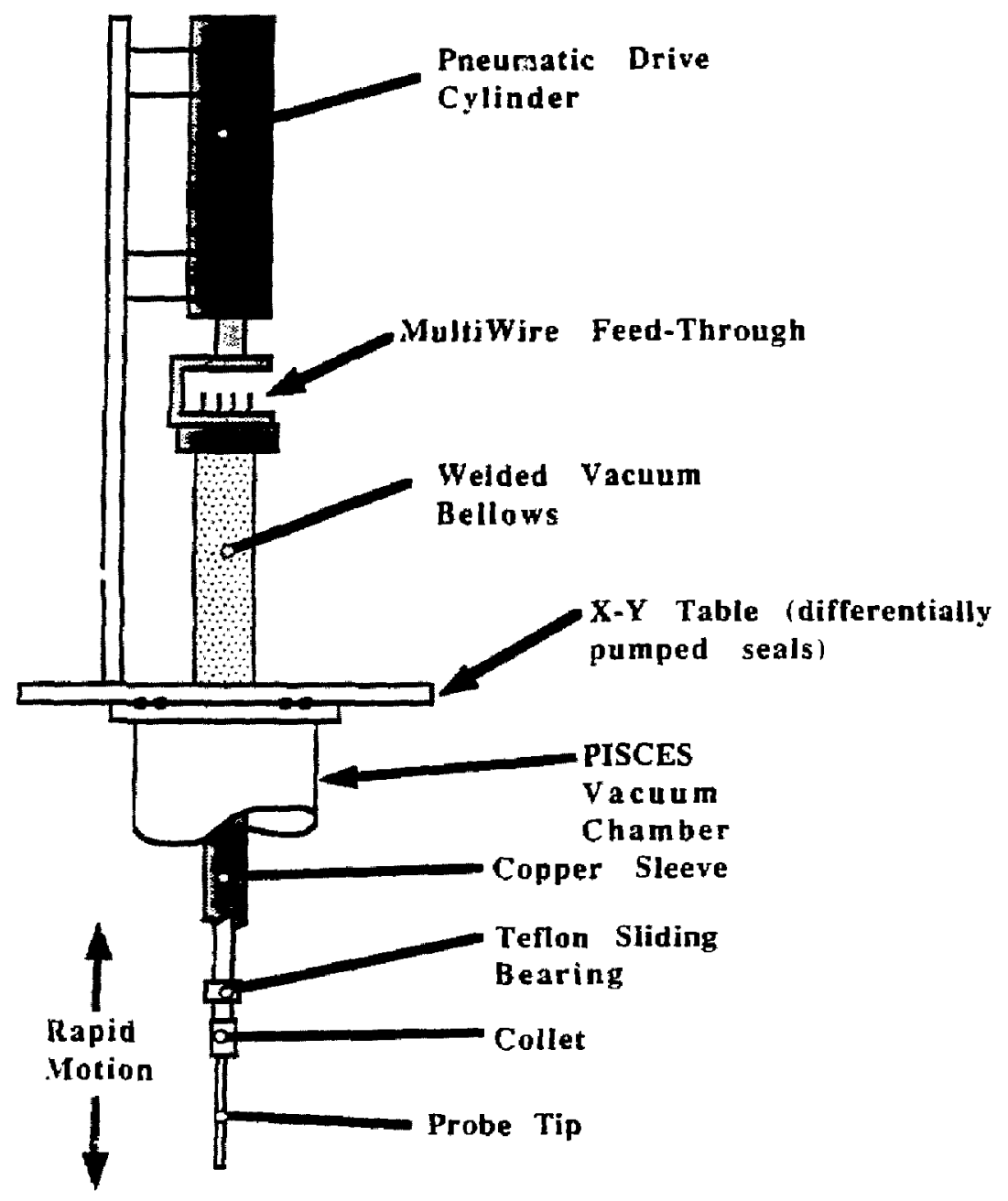

Figure 3 - Schematic diagram of the PISCES generic fast probe pneumatic actuato:. 


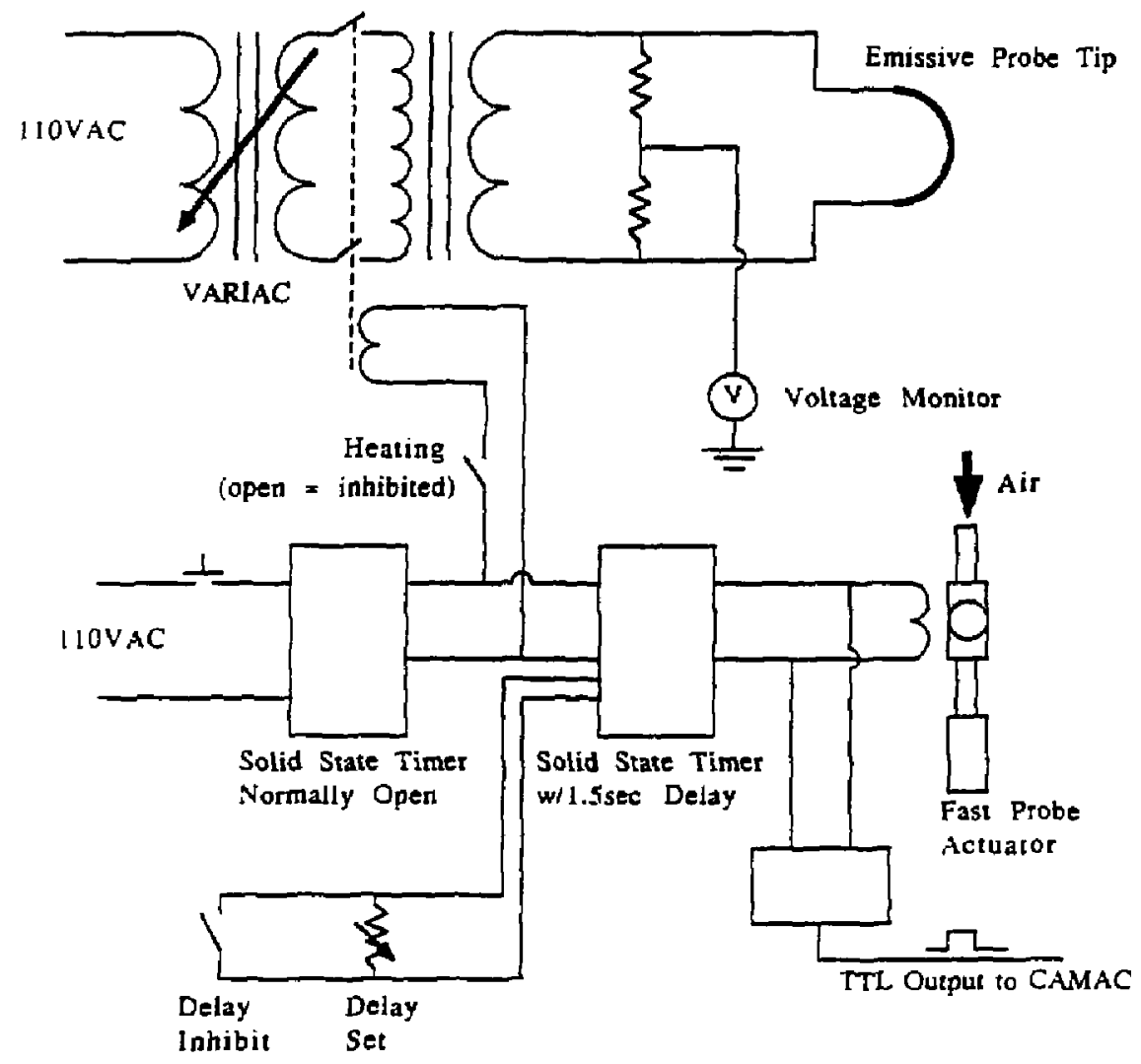

Figure 4 - Schematic of the emissive probe heating circuit and CAMAC trigger delay circuit. 


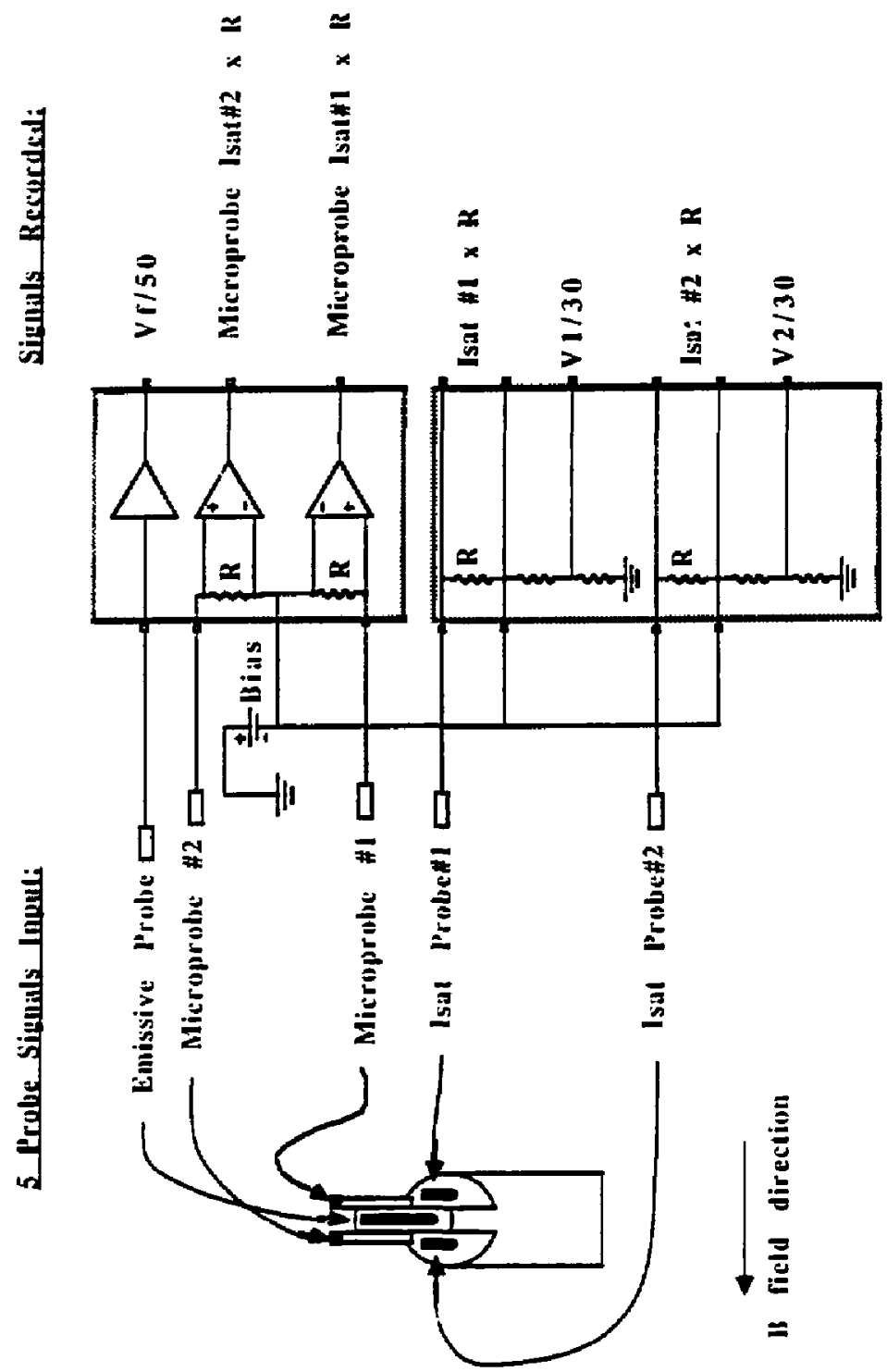

Figure 5 - Schematic of the data acquisition electronics for the emissive and mach probes. 


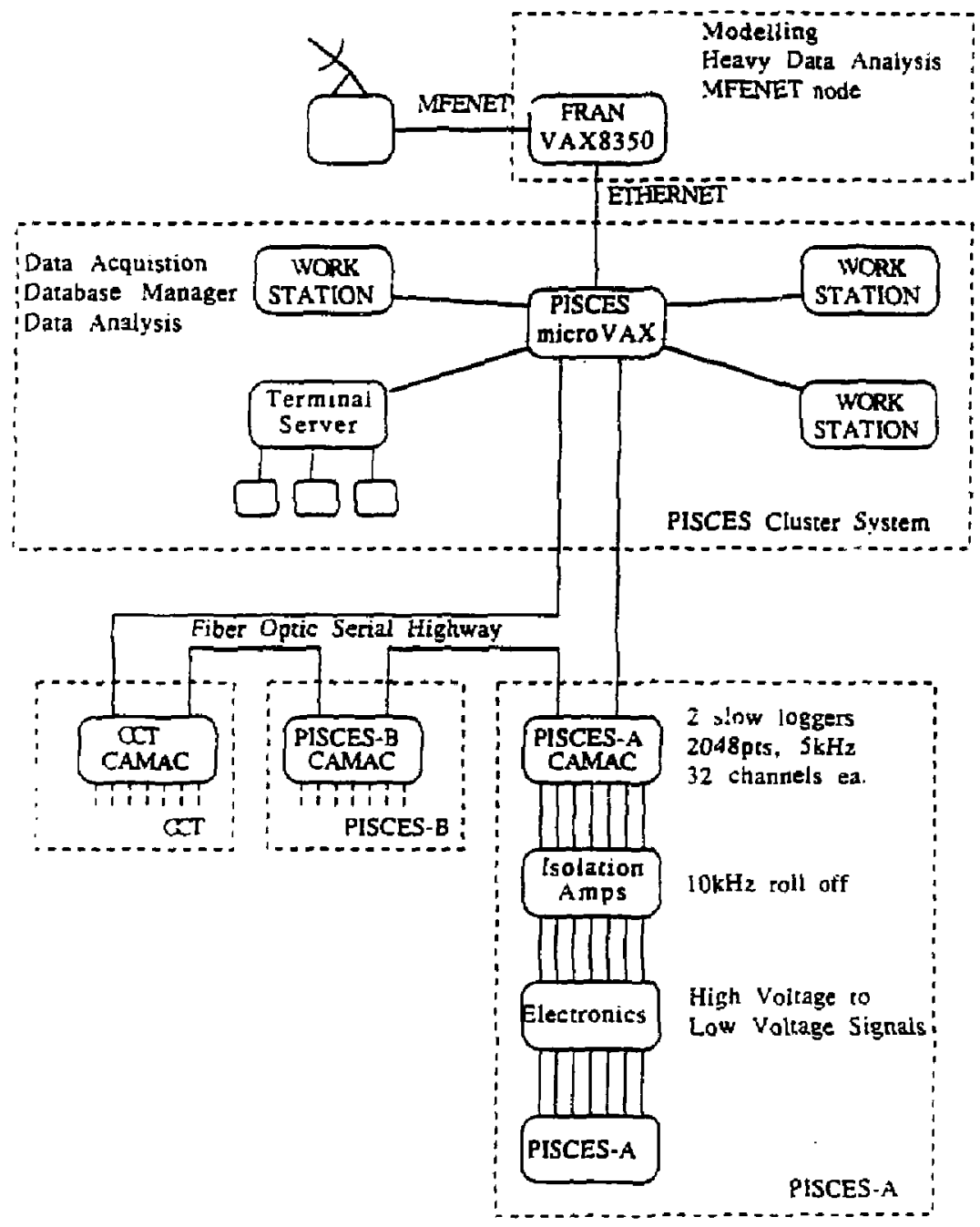

Figure 6 - PISCES data acquisition and analysis network showing the various functions performed by each computer node. 


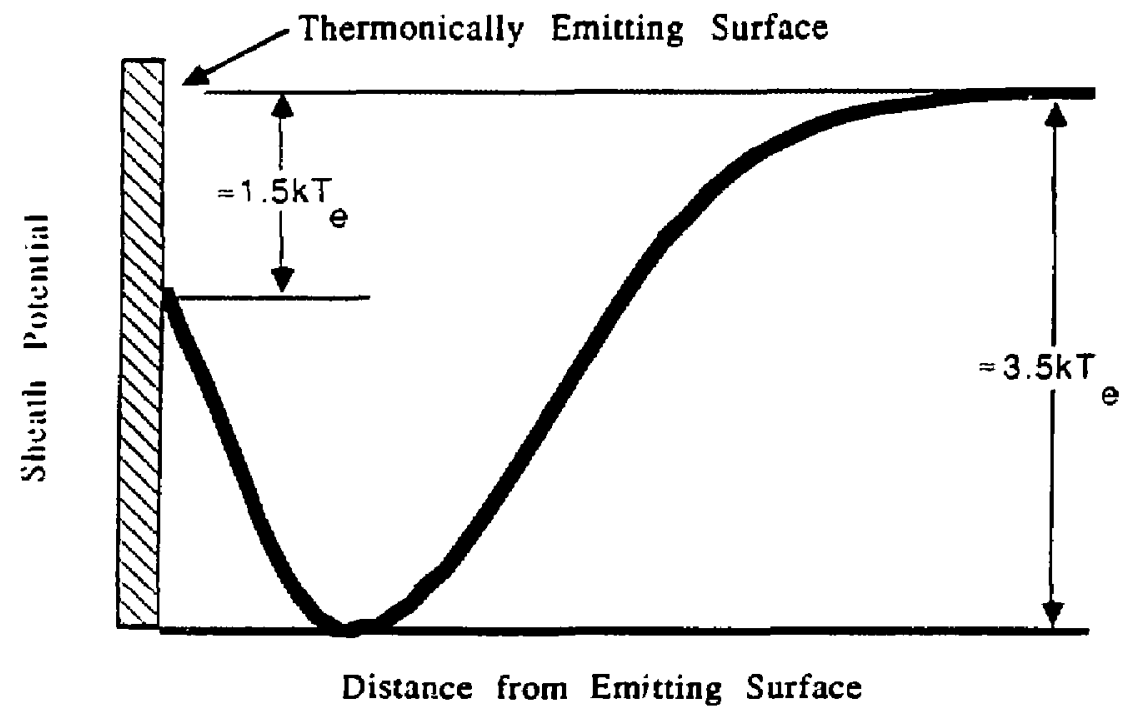

Figure 7 - The potential as a function of position within a double sheath; after F. F. Chen?. 


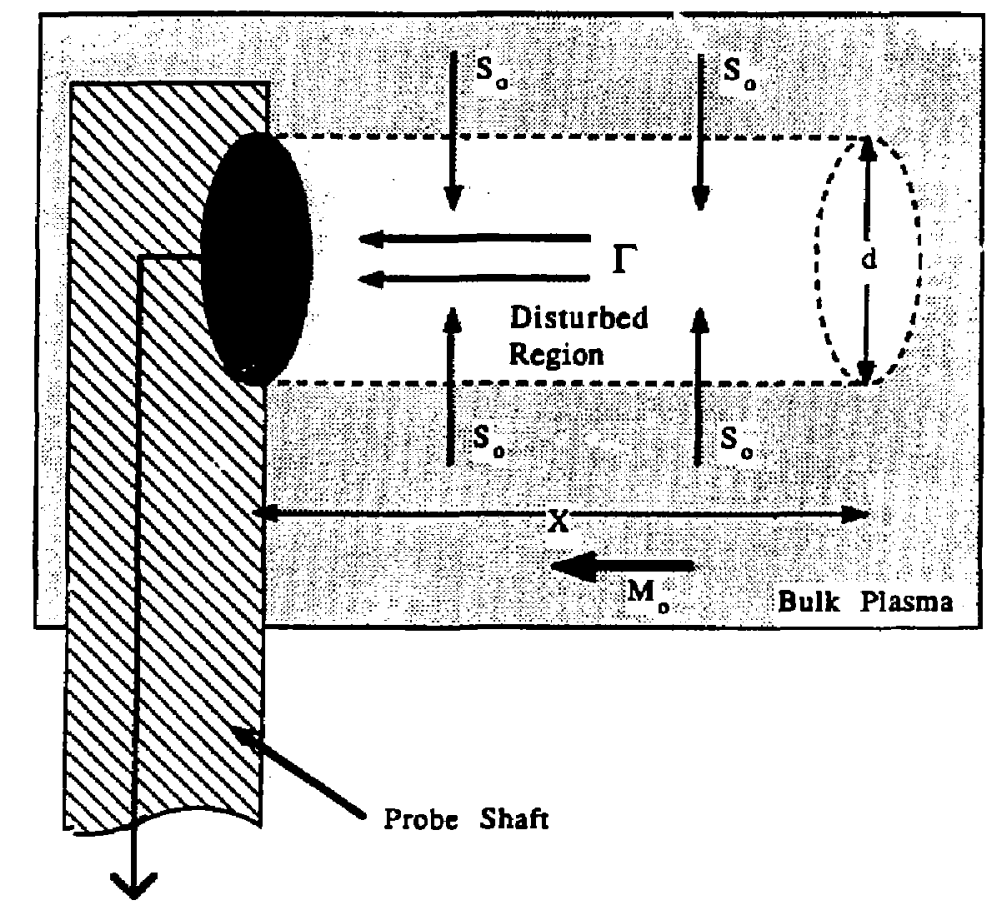

To probe electronics

Figure 8 - The source and sink of plasma in the flux tube of a mach probe tip according to the Stangeby model 11 . 


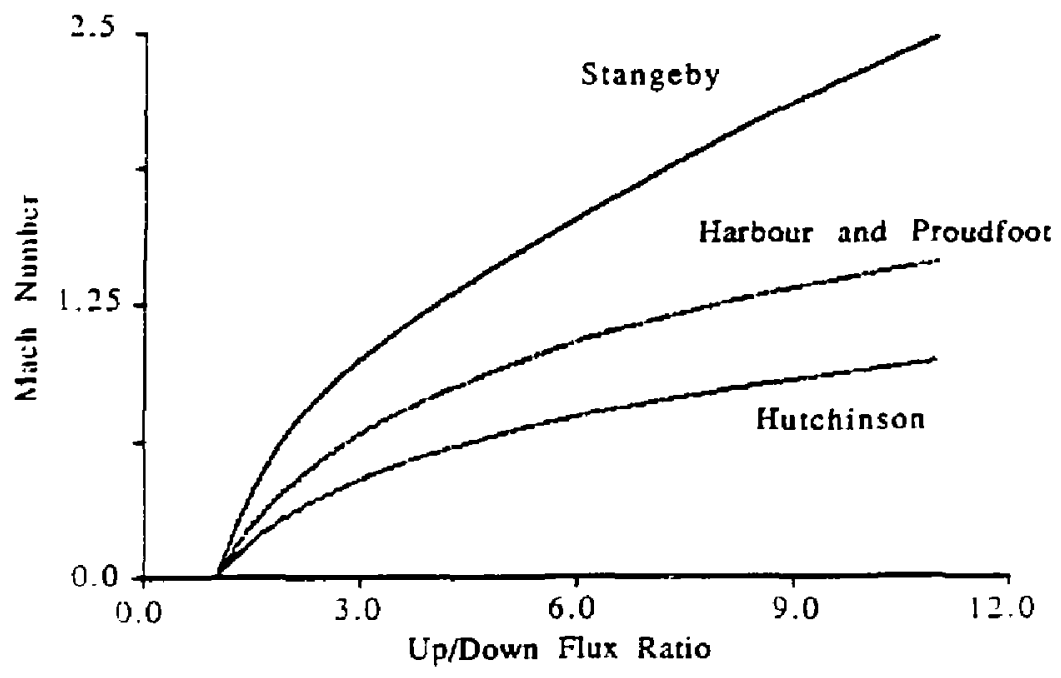

Figure 9 - Mach number computed using the Stangeby, Hutchinson, and Harbour and Proudfoot models versus upstream to downstream flux ratio. 

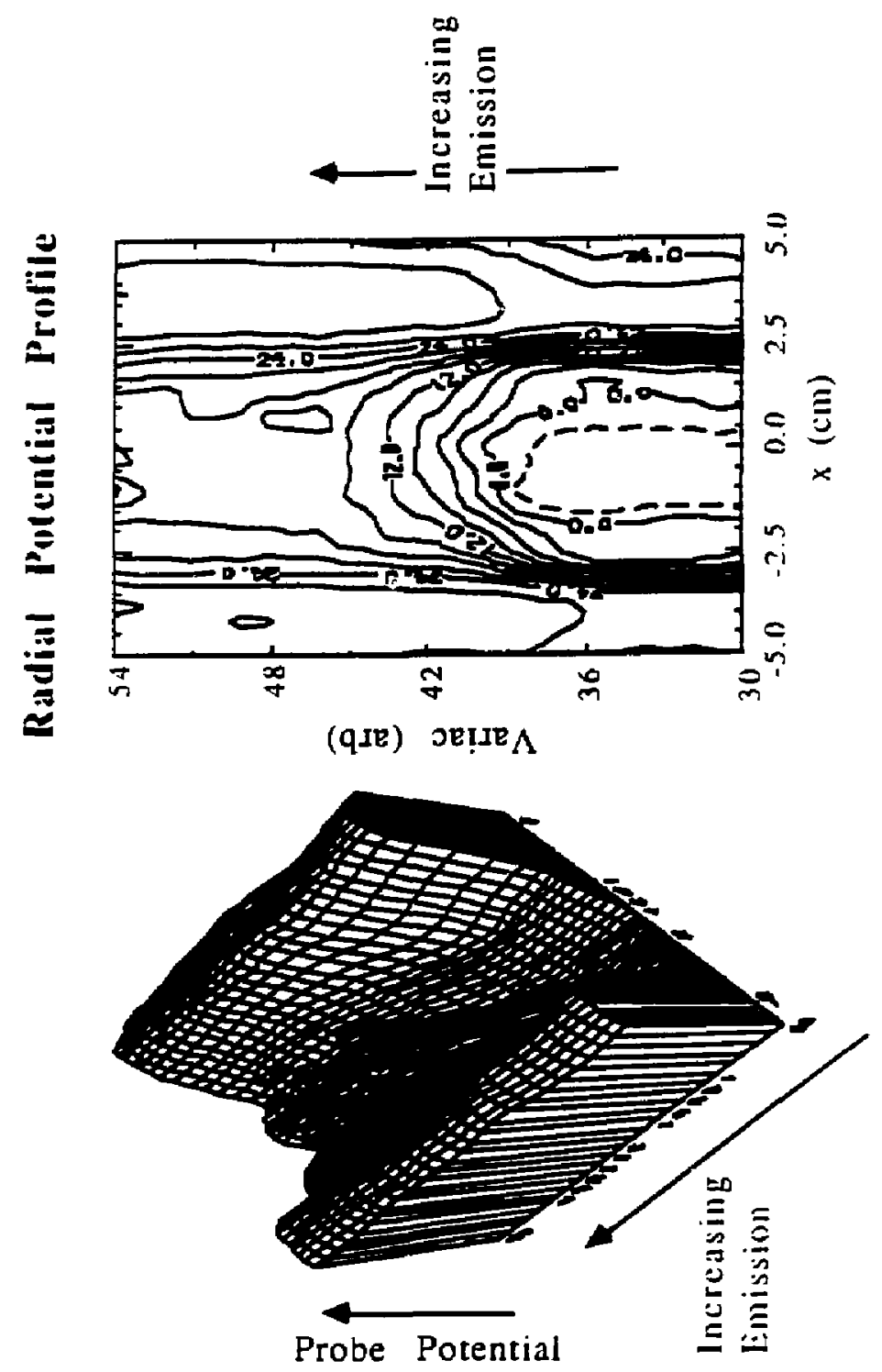

Figure 10 - Emitting probe potential profile and contour plot versus variac setting. 


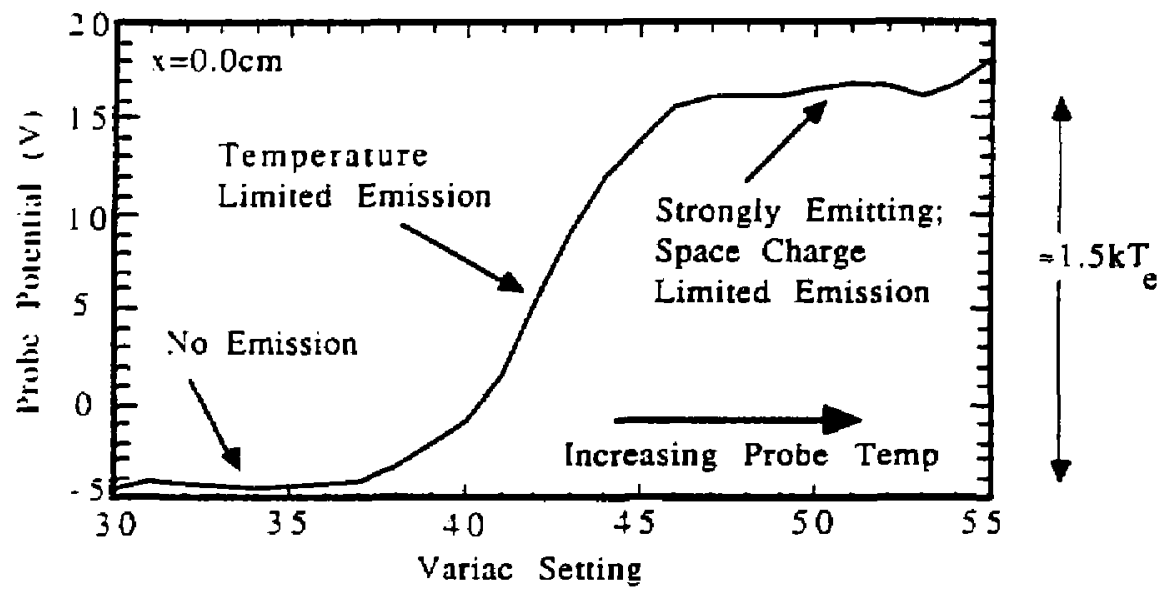

Figure 11 - Emitting probe potential versus variac setting at the center of the PISCES discharge. Observe regions of no emission, temperature limited emission, and space charge limited emission. 


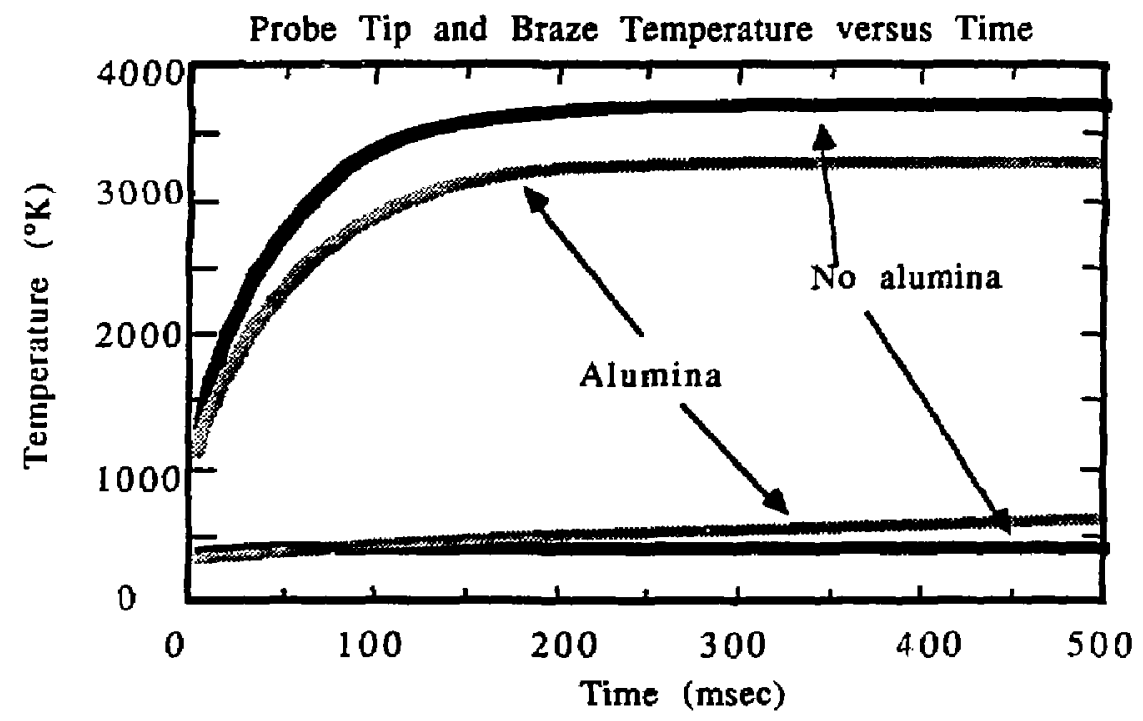

Figure 12 - Temperature versus time along tungsten tip and copper leads computed from one dimensional heat transfer equation in the absence and presence of the alumina insulator. Note temperature excursion undergone by braze joint without the alumina acting as heat reservoir. 


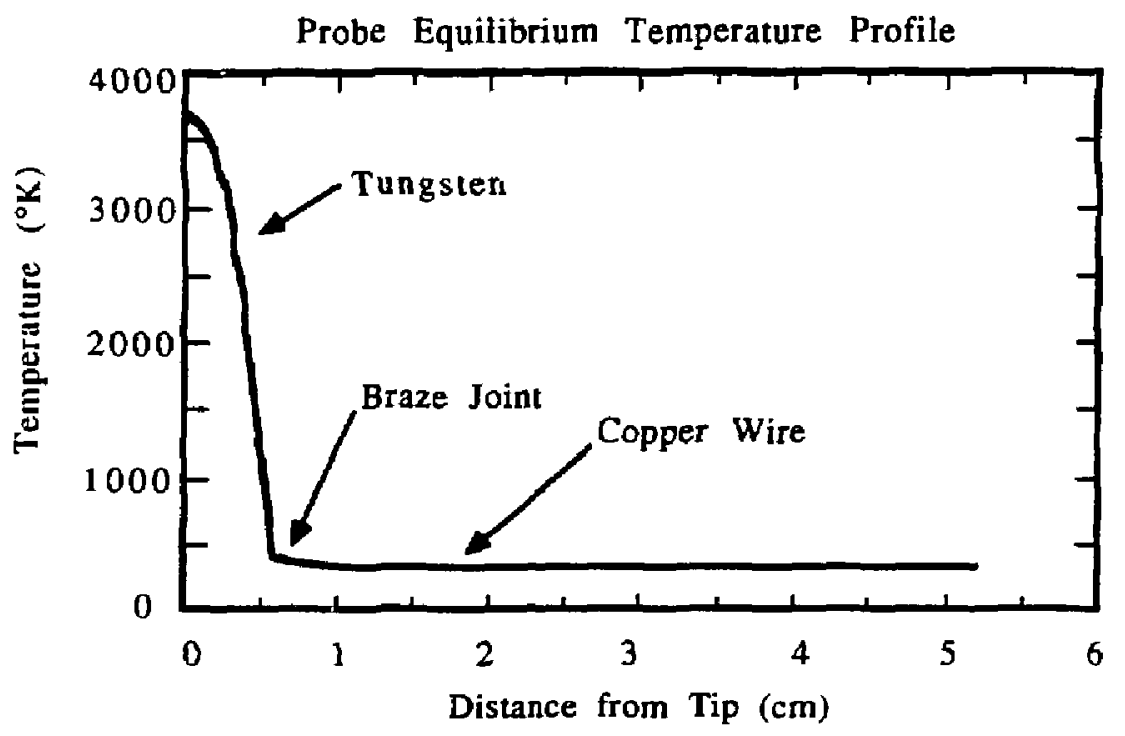

Figure 13 - Temperature versus position along tungsten tip and copper leads computed in the presence of alumina insulator acting as a heat reservoir. Note the steep temperature gradient in the tungsten filament near the braze joint. 


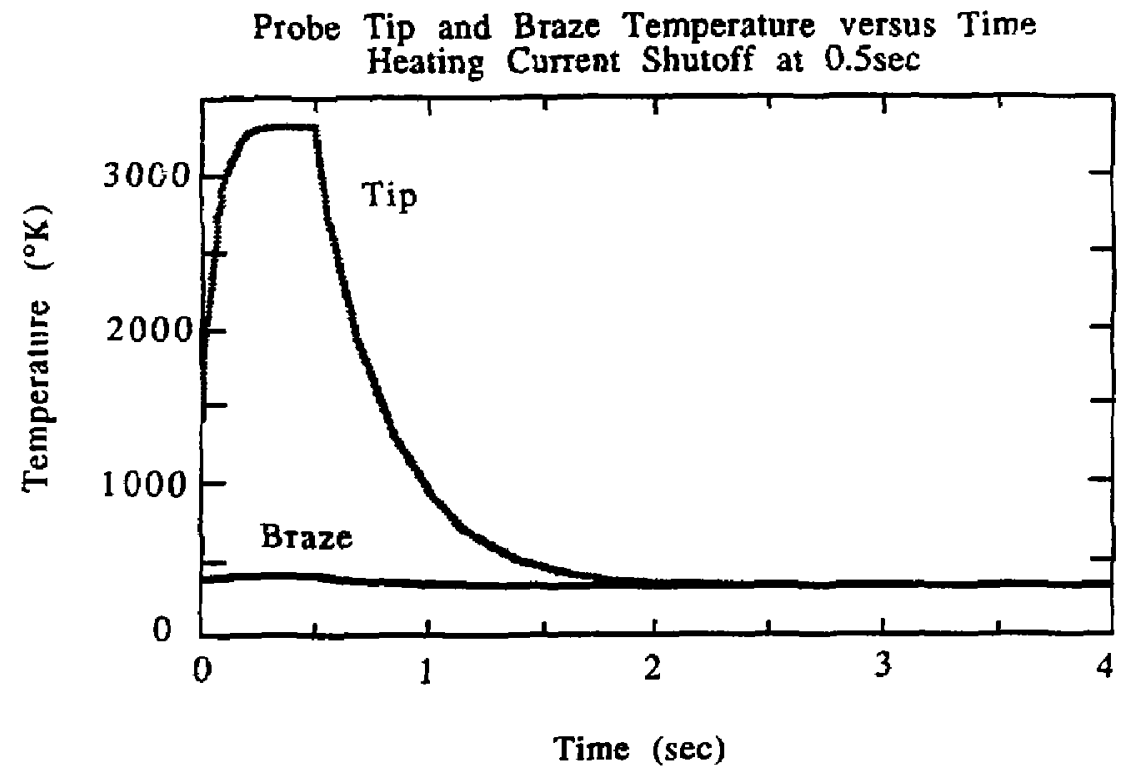

Figure 14 - Temperature versus time along tungsten tip and copper leads in presence of alumina insulator with heating current shut off at $0.5 \mathrm{sec}$. Copper leads show little overall temperature rise after 5 sec. 
Floating Potential Raw Data vs Position

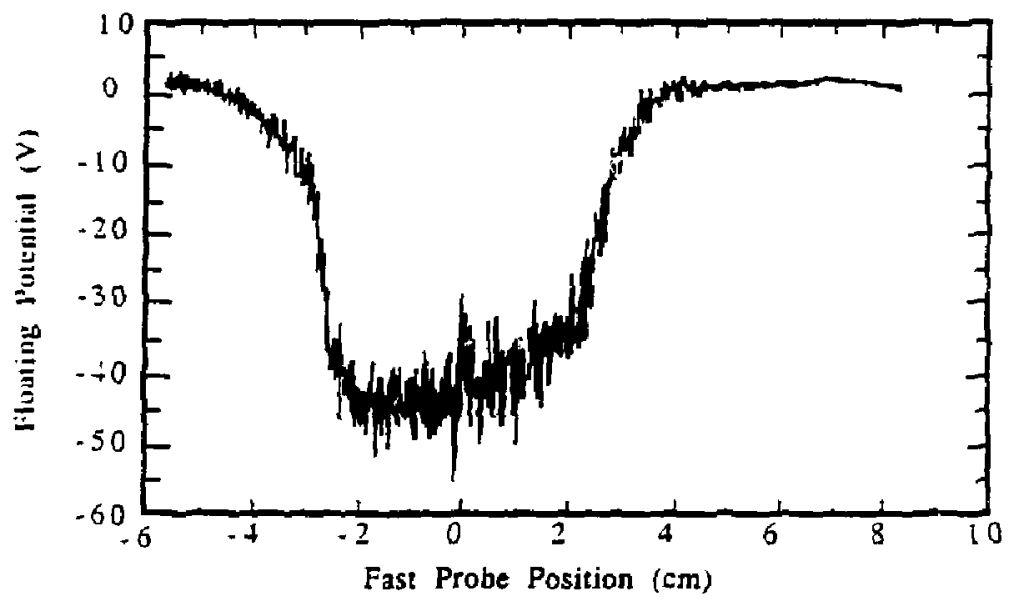

Space Potential Raw Data vs Position

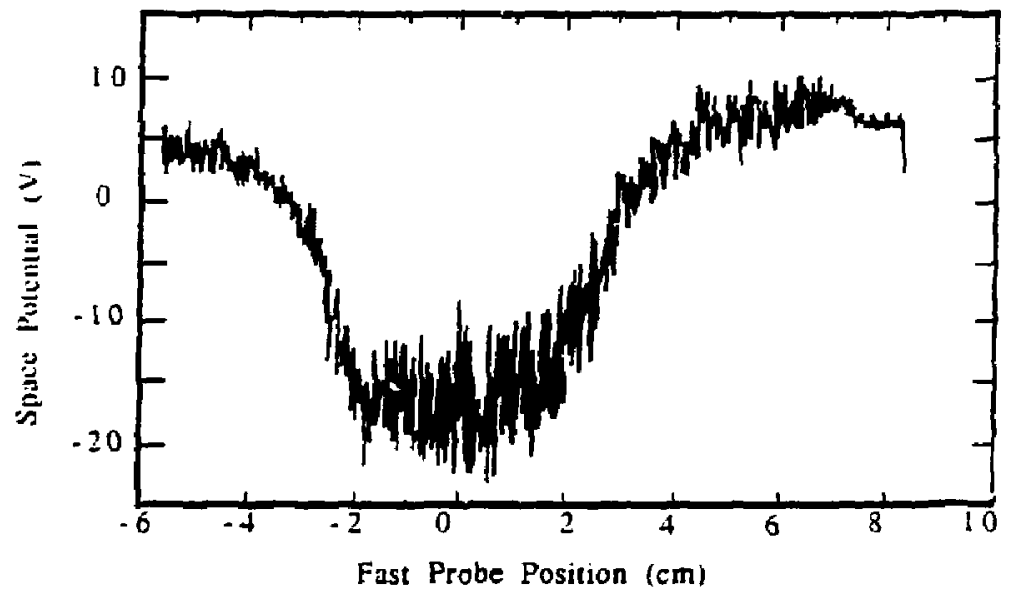

Figure 15 - Representative floating and space potential versus radial position profiles in PISCES using the scanning emissive probe diagnostic without heating emissive probe tip. Parabolic shape of potential profiles is indicative of reflex arc discharges. 

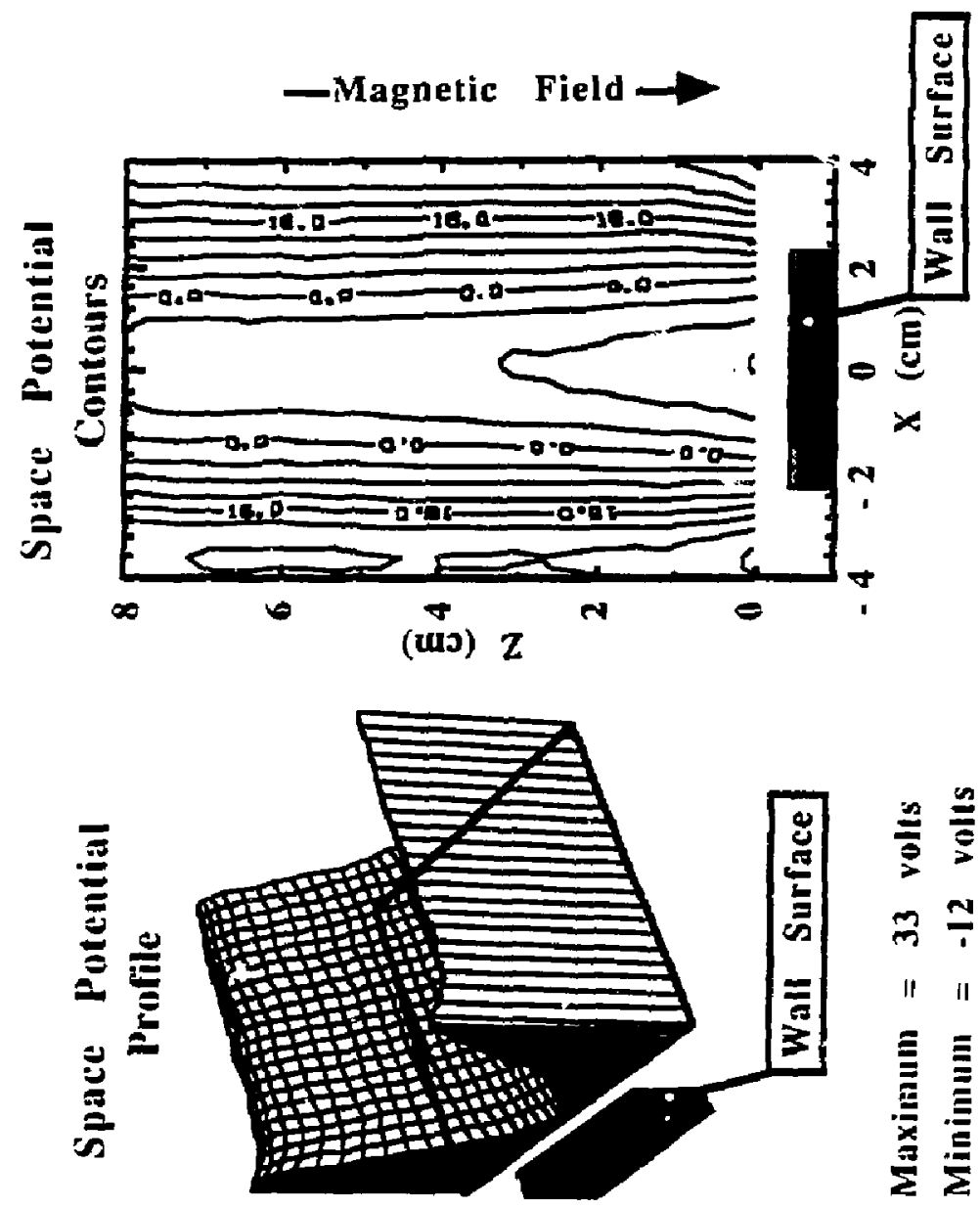

Figure 16 - PISCES Presheath Experiment data; three dimensional profile and contour plot of space potential. Note that the space potential is seen to vary by approximately $0.5 \mathrm{kT} e$ along the axis of experiment. 


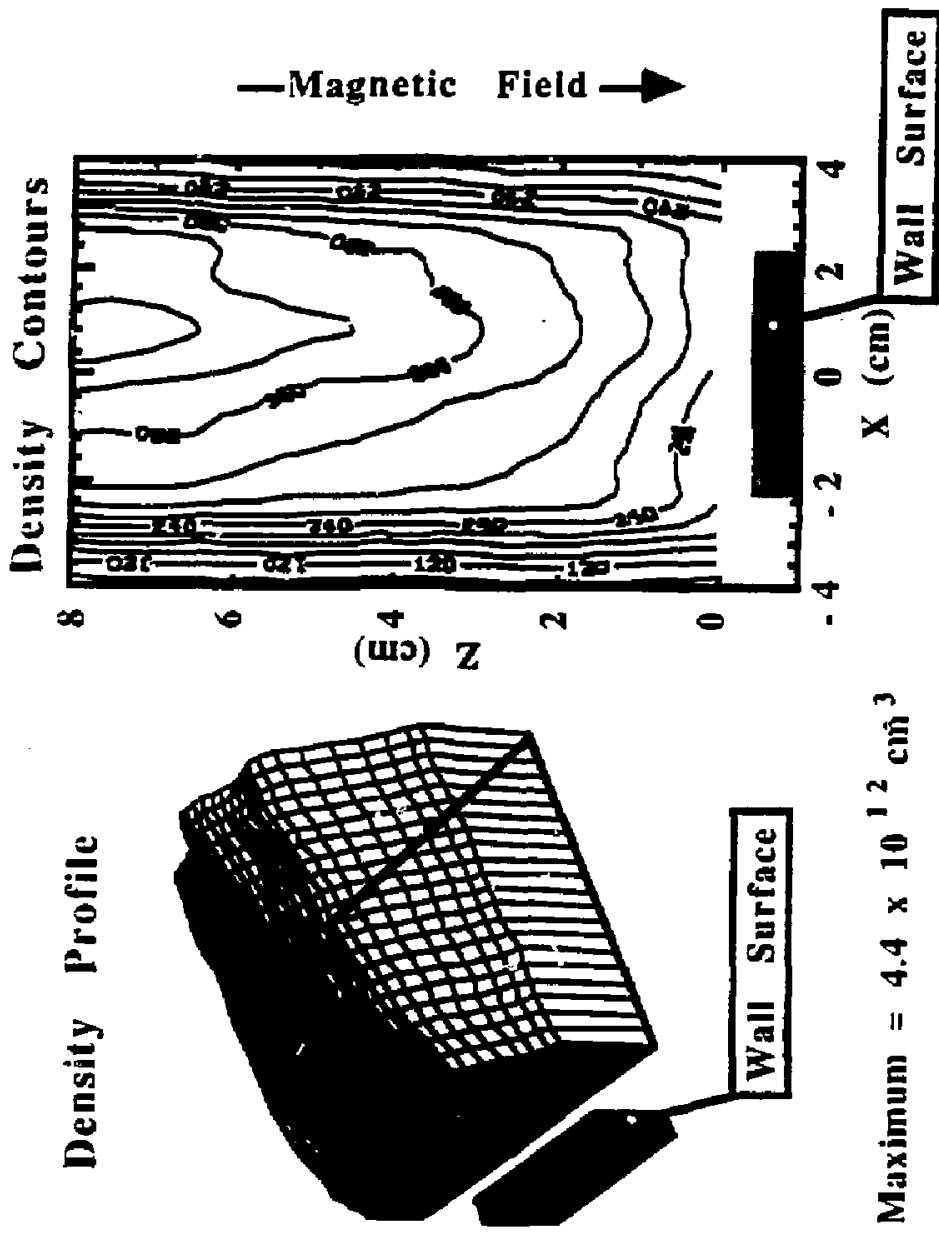

Figure 17 - PISCES Presheath Experiment data; three dimensional profile and contour plot of density as computed from average of flux collected by upstream and downstream mach probe. 

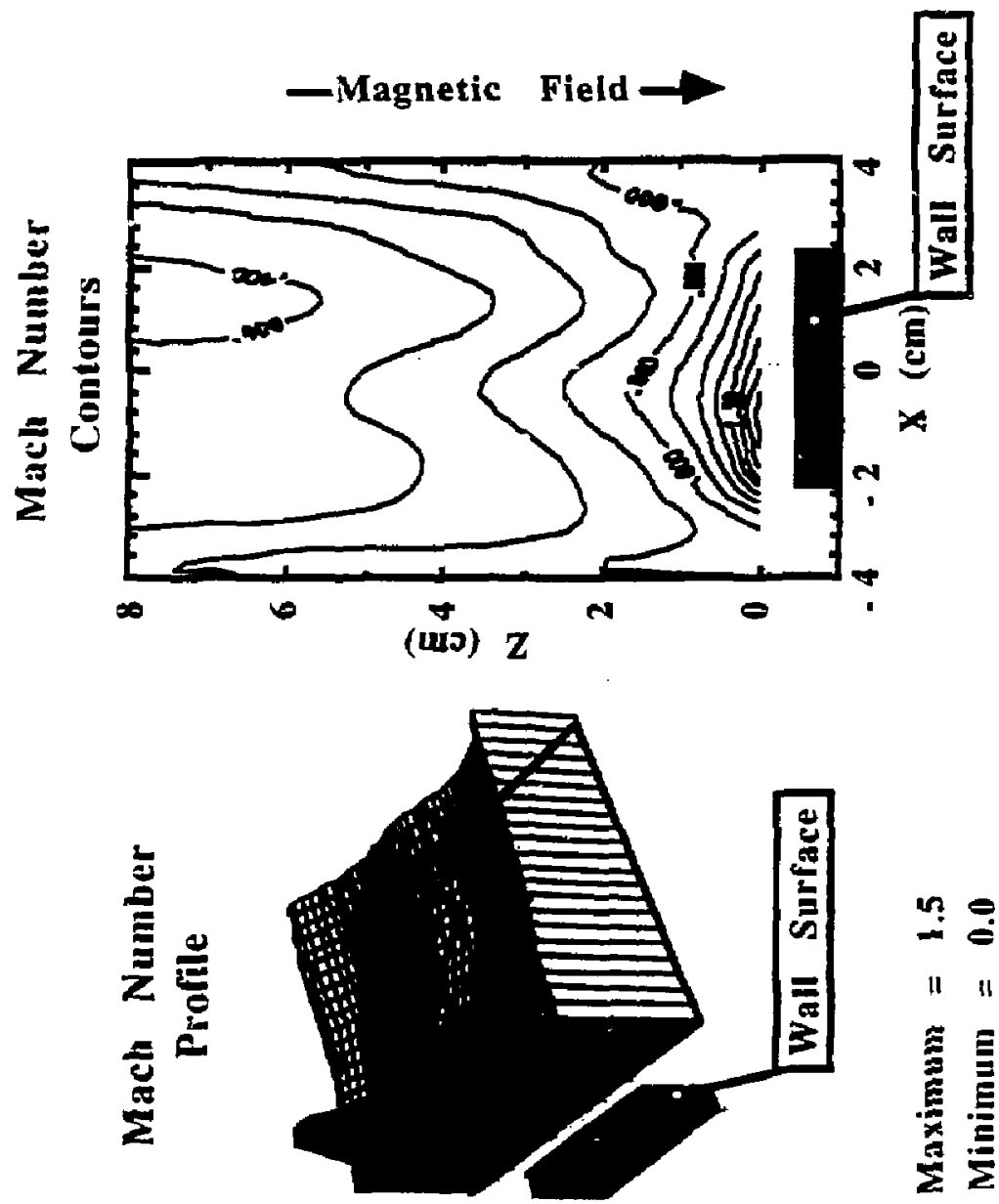

Figure 18 - PISCES Presheath Experiment data; three dimensional profile and contour plot of parallel mach number as computed by the Stangeby model. The computed mach number is actually greater than 1.0 near the surface, but the assumptions of the Stangeby model are violated because the surface is interrupting the downstream probe's flux tube. 


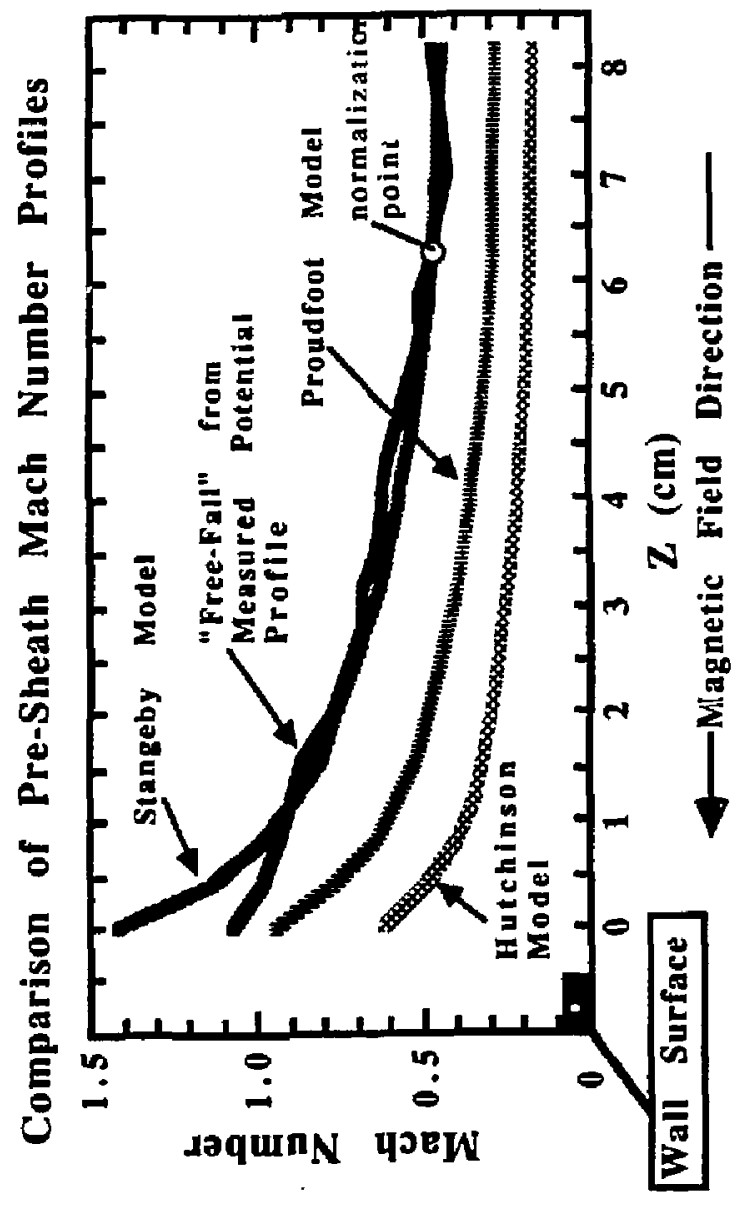

Figure 19 - Mach number computed from mach probe data via the Stangeby, Hutchinson, and Harbour and Proudfoot models and the mach number computed from the free fall model due to the measured space potential variation versus position along the axis of the discharge. The free fall model is normalized to the Stangeby mach number far upstream from the wall surface. 


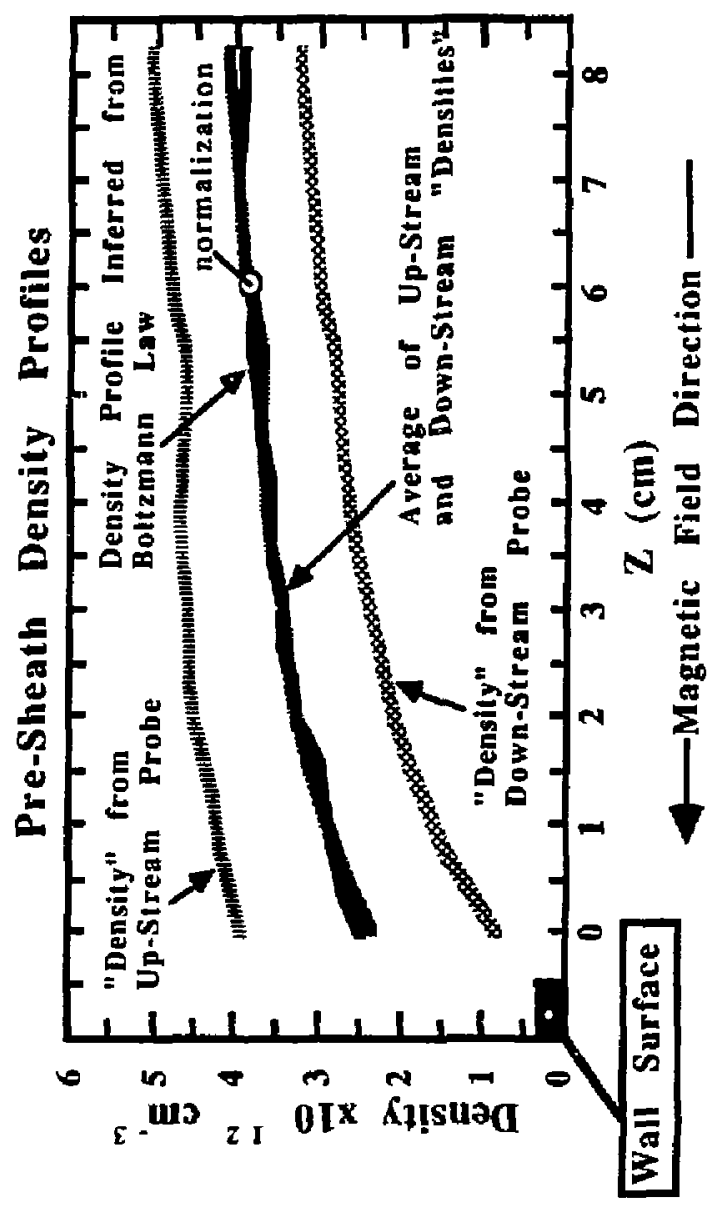

Figure 20 - Density as measured from the average of the upstream and downstream flux on the mach probes and the density computed from the Boltzmann Relation using the space potential variation measured by the emissive probe versus axial position along the center of the discharge. Density from the Boltzmann Relation is normalized to the measured density far upstream from the wall surface. 


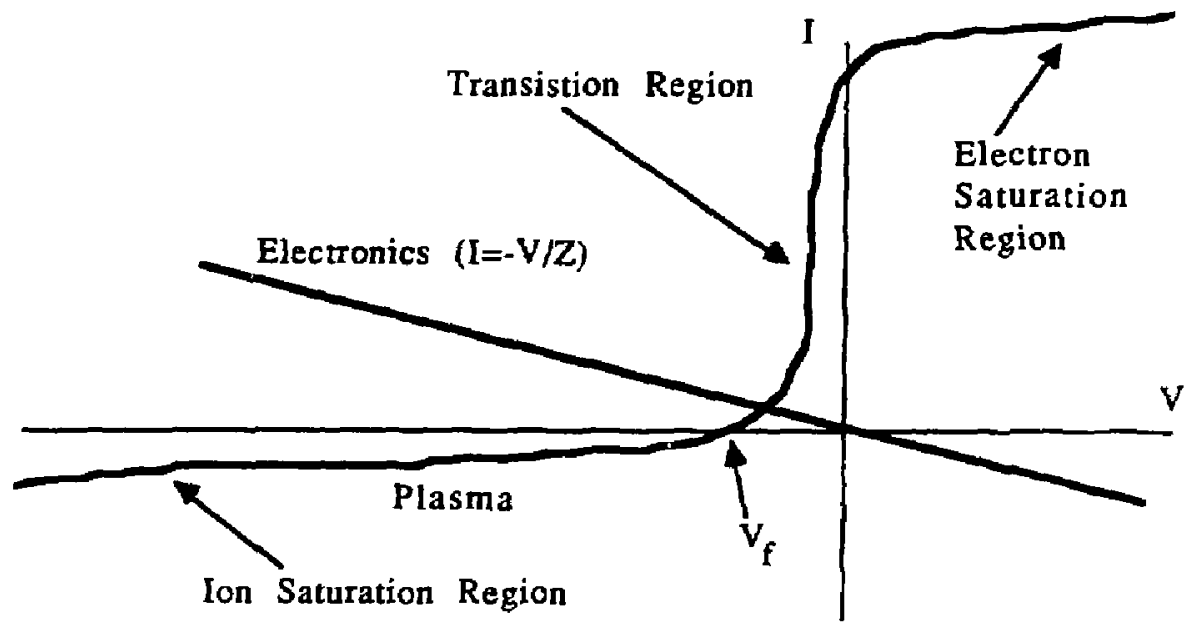

Figure 21 - A typical I-V characteristic of a cold probe in a plasma and the load line of the measuring electronics with finite impedance. 


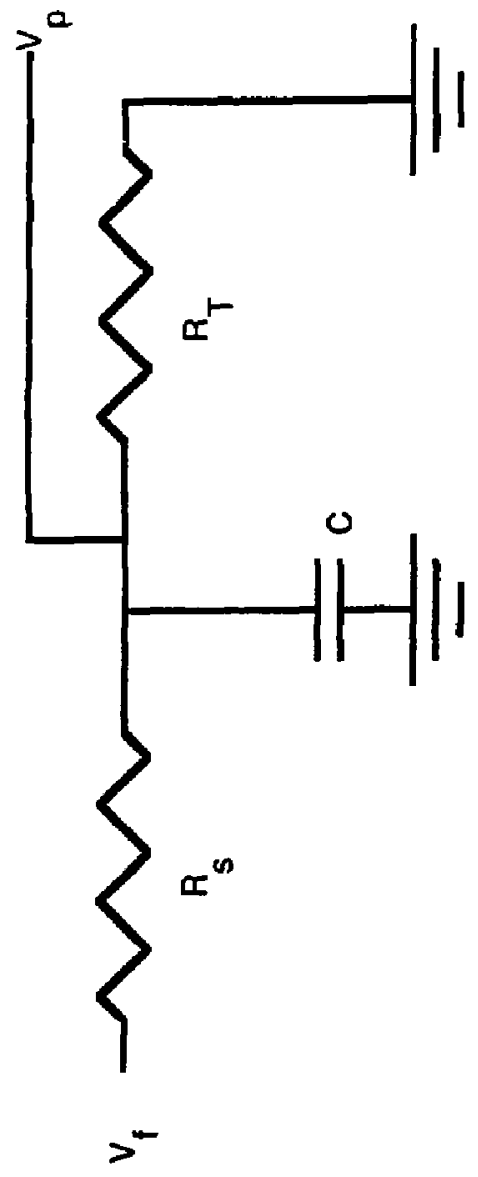

Figure 22 - A simple network modelling the effect of the plasma source impedance, stray cable capacitance, and finite input impedance of the data acquisition electronics. 


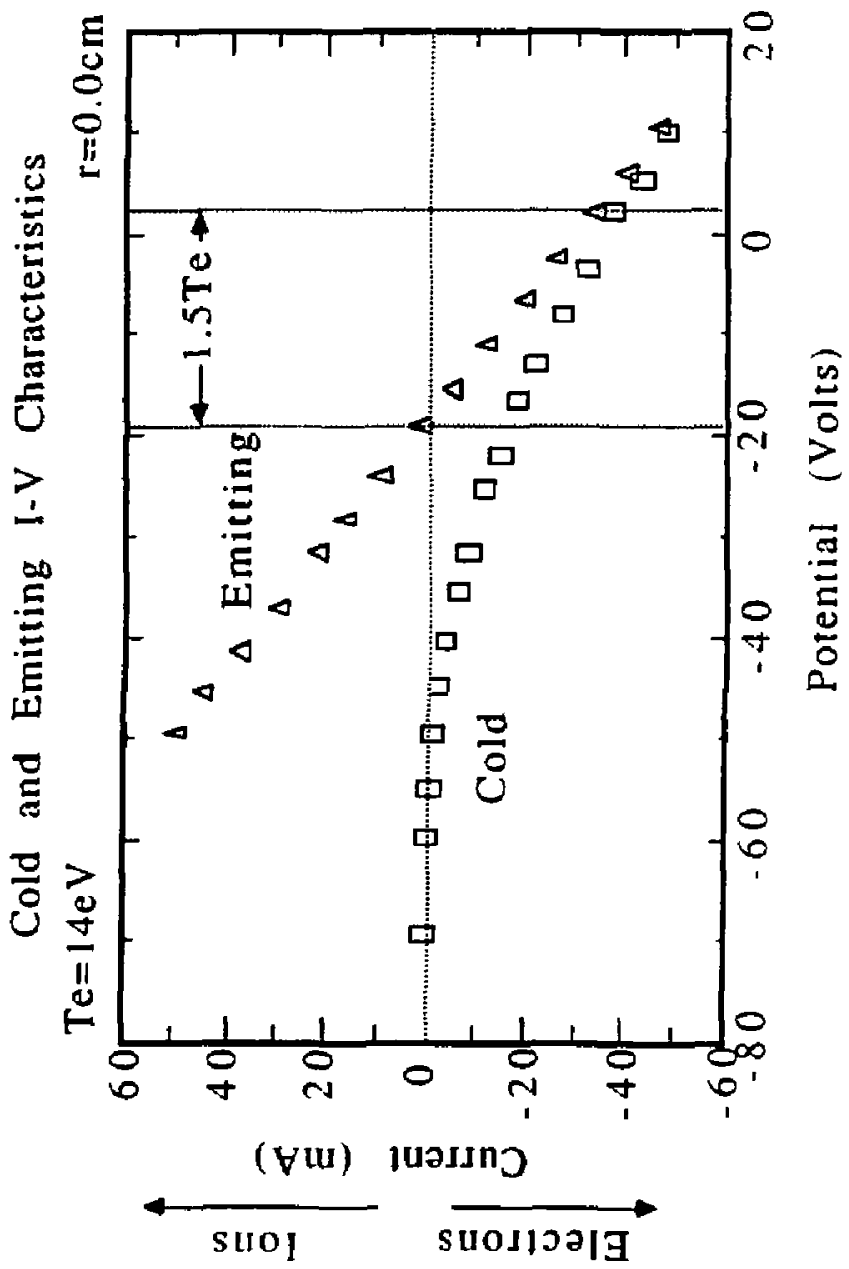

Figure 23 - I-V characteristics of the emissive probe when cold and emitting at the center of the discharge. Observe that the floating potential of the emitting probe is $1.5 \mathrm{kT}_{e}$ below the true space potential at the divergence of the two characteristics. 


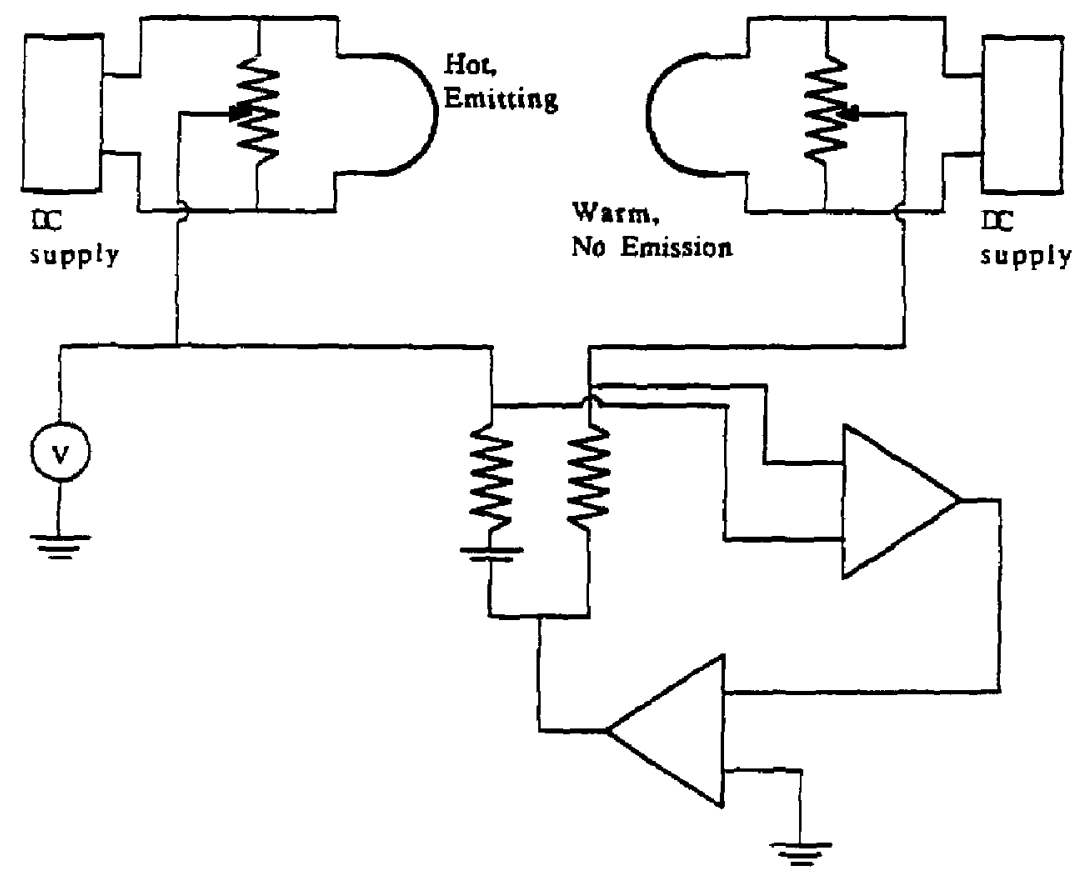

Figure 24 . Schematic diagram of the differential amplifier and feedback circuit necessary for the differential emissive probe. 